\title{
Über das Holomorphiegebiet der Vierpunkt-Funktion
}

\author{
E. BRÜNING \\ Institut für Theoretische Physik, Universität Würzburg
}

Eingegangen am 3. Juni 1971

\begin{abstract}
A new method of constructing analytic continuations of matrixelements of fieldoperators is presented for the example of the fourpoint-functions whose boundary values generate a double commutator. Writing the Wightman-functions as matrixelements of the translationoperator we get an integral representation of these functions over absolutely integrable complex-valued measures which depend analytically on the variables of the Wightman-functions. By controlling the growth-properties of the analytic continuation of these measures we end up with an integral representation of the analytic continuation of these four Wightman-functions into an $\mathscr{L}^{+}$-invariant domain of holomorphy which connects the original four tubes.
\end{abstract}

\section{Einleitung}

Die ursprünglichen Absichten der Wightman-Formulierung einer Feldtheorie ([13], p. 72, [20]) ließen sich bisher nicht vollständig verwirklichen. Dennoch bleibt das Problem der analytischen Fortsetzung von Erwartungswerten von Feldoperatoren interessant. In einem direkteren Zusammenhang mit meßbaren Größen als die Wightman-Funktionen stehen Streumatrixelemente, die sich als Fouriertransformierte von vollständig retardierten Kommutatoren schreiben lassen [10]. So ist heute das Problem der analytischen Fortsetzung von Erwartungswerten von Feldoperatoren vor allem im Zusammenhang mit dem Beweis der Analytizitätseigenschaften von Streumatrixelementen aktuell, und zwar auch in der Theorie der lokalisierten Observablen $([1-3,7,9])$.

Am Beispiel der vier Vierpunkt-Wightman-Funktionen, deren Randwerte gerade den doppelten Kommutator von vier skalaren WightmanFeldern erzeugen, demonstrieren wir eine neue Methode zur analytischen Fortsetzung von Vakuumerwartungswerten von Feldoperatoren.

Im Prinzip besteht diese Methode aus folgenden Schritten:

1. Wir schreiben die Wightman-Funktionen als Matrixelemente des Translationsoperators $T(x)=U(x, 1)$ zwischen geeigneten Zuständen $((a, \Lambda) \rightarrow U(a, \Lambda)$ sei die unitäre Darstellung der orthochronen eigentlichen Lorentzgruppe $\mathscr{L}_{+}^{\uparrow}$, die gemäß den Wightman-Axiomen im Hilbertraum $\mathfrak{S}$ der Zustände unserer Theorie existiert). 
2. Aus 1. folgt leicht eine Darstellung der Wightman-Funktionen als Integrale über absolutintegrierbare komplexwertige Maße. Diese Maße hängen analytisch von den Variablen der Wightman-Funktionen $a b$ und sind in einem viel größeren Gebiet als diese analytisch.

3. Die Lokalität der Theorie impliziert die Existenz einer gemeinsamen analytischen Fortsetzung der in 2. eingeführten Maße.

4. Der entscheidende Schritt dieser Methode besteht nun darin, aus der Kenntnis des Wachstums dieser Maße in den Tuben das Wachstum der gemeinsamen analytischen Fortsetzung dieser Maße in ihrem Holomorphiegebiet zu erschließen.

5. Die Kenntnis der Wachstumseigenschaften der analytischen Fortsetzung der Maße erlaubt, eine gemeinsame analytische Fortsetzung der Wightman-Funktionen als Integral über die gemeinsame analytische Fortsetzung der Maße anzugeben. Das so erhaltene Holomorphiegebiet der Wightman-Funktionen wird durch die Wachstumseigenschaften der Maße bestimmt.

Unmittelbar ist keineswegs klar, ob auf diese Weise eine echte Erweiterung des ursprünglichen Holomorphiegebietes resultiert. Das explizit durchgeführte Beispiel der Vierpunkt-Funktionen jedoch zeigt, daß die obige Methode eine nichttriviale Erweiterung des ursprünglichen Holomorphiegebietes (die vier Tuben) liefern kann.

Darüber hinaus bestehen folgende Hoffnungen:

a) Diese Methode sollte auf alle höheren $n$-Punkt-Funktionen übertragbar sein.

Die Schritte 1., 2. und 3. lassen sich ohne weitere Schwierigkeiten durchführen. Bisher erscheinen die Komplikationen im 4. und 5. Schritt, die insbesondere in der größeren Anzahl der Variablen bestehen, überwindbar.

b) Die bisherigen Untersuchungen des Holomorphiegebietes der $n$-Punkt-Funktionen benutzten die aus der $\mathscr{L}_{+}^{\uparrow}$-Invarianz dieser Funktionen folgende Darstellung durch Funktionen der Invarianten bezüglich $\mathscr{L}_{+}(\mathbb{C})$. Die Gesamtzahl

$$
r=\left(\begin{array}{c}
n+1 \\
2
\end{array}\right)+\left(\begin{array}{l}
n \\
4
\end{array}\right)
$$

dieser Invarianten ist für $n=5$ gleich der Anzahl der Vektorvariablen $\left(\zeta_{1} \ldots, \zeta_{n}\right)$ und für $n>5$ größer als die Anzahl der Vektorvariablen. Außer durch die größere Anzahl der Variablen für $n>5$ wird die Untersuchung des Holomorphiegebietes in den Invarianten dadurch erschwert, daß die Invarianten nicht unabhängig voneinander sind. Diese beiden Nachteile können mit der oben angedeuteten Methode vermieden werden. Damit eröffnet sich eine Möglichkeit, Fortschritte im Sinne der ursprünglichen Absichten der Wightman-Theorie zu erzielen. 
c) Bei den niedrigen $n$-Punkt-Funktionen besteht eine Symmetrie im Analytizitätsverhalten im Orts- und Impulsraum. Daher scheint diese Methode eine neue Möglichkeit zur Untersuchung von Streumatrixelementen zu eröffnen. Im Hinblick auf solche Untersuchungen wurde das explizit durchgeführte Beispiel gewählt.

Bei der Durchführung dieser Methode für die Vierpunkt-Funktionen wollen wir die Postulate der Theorie eines skalaren Wightman-Feldes $([13,17])$ zugrundelegen, halten es aber für sehr wahrscheinlich, daß weite Teile der Argumentation auch in einer Theorie lokalisierter Observablen richtig bleiben.

In der Bezeichnungsweise von [13] untersuchen wir die vier Vierpunkt-Wightmanfunktionen, deren Randwerte gerade den doppelten Kommutator

$$
\begin{aligned}
\left(\Omega_{0},\right. & {\left.\left[A_{1}\left(x_{1}\right), A_{2}\left(x_{2}\right)\right]\left[A_{3}\left(x_{3}\right), A_{4}\left(x_{4}\right)\right] \Omega_{0}\right) } \\
= & \mathfrak{W}_{1234}\left(x_{1}, x_{2}, x_{3}, x_{4}\right)-\mathfrak{W}_{2134}\left(x_{2}, x_{1}, x_{3}, x_{4}\right) \\
& \quad-\mathfrak{W}_{1243}\left(x_{1}, x_{2}, x_{4}, x_{3}\right)+\mathfrak{W}_{2143}\left(x_{2}, x_{1}, x_{4}, x_{3}\right)
\end{aligned}
$$

erzeugen $\left(\Omega_{0}\right.$ bezeichne hier den Vakuumzustand im Hilbertraum $\mathfrak{H}$ unserer Theorie, $A_{j}(x)$ seien vier skalare Wightman-Felder). Indem wir die $W$-Funktionen als Matrixelemente des Translationsoperators zwischen geeigneten Zuständen aus $\mathfrak{H}$ aufschreiben, gelingt es leicht, eine einfache Integraldarstellung der $W$-Funktionen über Funktionen anzugeben, die in einem viel größeren Gebiet als die $W$-Funktionen analytisch sind (Abschnitt II). Die Lokalität impliziert die Existenz einer gemeinsamen analytischen Fortsetzung dieser Integralkerne, deren Wachstum in den vier Tuben bekannt ist (Abschnitt IV). Im III. Abschnitt stellen wir Mittel zur Kontrolle des Wachstums dieser gemeinsamen analytischen Fortsetzung in ihrem gesamten Holomorphiegebiet bereit. Damit kann schließlich im Abschnitt IV eine gemeinsame analytische Fortsetzung der vier $W$-Funktionen als Integral über die analytische Fortsetzung der Integralkerne konstruiert werden. Ohne explizit die $\mathscr{L}_{+}^{\uparrow}$-Invarianz der Theorie auszunutzen, erhalten wir so ein $\mathscr{L}_{+}^{\uparrow}$-invariantes Holomorphiegebiet $\Omega$, welches die vier Tuben enthält und eine eindeutige analytische Fortsetzung der vier $W$-Funktionen nach $\Omega$.

\section{Eine Integraldarstellung für Wightman-Funktionen}

Zunächst geben wir ein paar einleitende Anmerkungen über die analytische Fortsetzung des Translationsoperators. Nach dem SNAGTheorem [6] läßt sich jede unitäre stetige Darstellung der Translationsgruppe $x \rightarrow T(x)$ als ein Operator-Stieltjes-Integral über ein eindeutig

17 Commun. math. Phys., Vol. 23 
durch $T(x)$ bestimmtes Spektralmaß $d E(p)$ auf $\mathbb{R}^{4}$ schreiben:

$$
\begin{aligned}
T(x) & =\int e^{i(x, p)} d E(p) \\
P & =\left(p^{0}, p^{1}, p^{2}, p^{3}\right) \\
p^{\mu} & =\int q^{\mu} d E(q), \quad \mu=0,1,2,3
\end{aligned}
$$

wird als Energie-Impuls-Operator interpretiert. Die Spektrumsbedingung gilt genau dann, wenn das Spektralmaß $d E(p)$ seinen Träger im abgeschlossenen Vorwärts-Lichtkegel $\overline{V^{+}}$hat. Ist nun $f$ eine bezüglich $d E(p)$ meßbare und integrierbare Funktion, so definiert das OperatorStieltjes-Integral $\int f(q) d E(q)$ einen beschränkten Operator $f(P)$ auf $\mathfrak{H}$ mit der Eigenschaft

$$
(\Phi, f(P) \Psi)=\int_{V^{+}} f(q) d(\Phi, E(q) \Psi)
$$

für alle $\Phi, \Psi \in \mathfrak{H}$.

Wir nennen eine auf einen Bereich $B \subset \mathbb{C}^{n}$ definierte Funktion $A(z)$ mit Werten, die Operatoren im Hilbertraum $\mathfrak{H}$ sind, analytisch, wenn für alle $\Phi \in \mathfrak{H}$ die Matrixelemente $(\Phi, A(z) \Phi)$ analytische Funktionen auf $B$ sind. Sei $\chi$ eine stetige beschränkte Funktion, so ist $\chi$ insbesondere bezüglich $d E(p)$ meßbar und integrierbar; dies gilt auch für $e^{i(z, q)} \chi(q)$ für $z \in \overline{\mathscr{T}^{+}}=\mathbb{R}^{4}+i \overline{V^{+}}$. Folglich ist

$$
\hat{T}_{\chi}(z):=\int_{V^{+}} e^{i(z, q)} \chi(q) d E(q)
$$

für alle $z \in \overline{\mathscr{T}}^{+}$ein wohldefinierter beschränkter Operator auf $\mathfrak{H}$. Über die z-Abhängigkeit dieses Operators gibt der folgende Satz nähere Auskunft:

Satz II.1. Ist $x \rightarrow T(x)$ eine stetige unitäre Darstellung der Translationsgruppe des Minkowskiraumes, so ist der für stetige, beschränkte Funktionen $\chi$ durch (II.1) definierte Operator $\hat{T}_{\chi}(z)$ eindeutig durch $T(x)$ bestimmt und hat folgende Eigenschaften:

(1) $\hat{T}_{\chi}(z)$ ist stetig in $z \in \overline{\mathscr{T}}^{+}$,

(2) $\hat{T}_{x}(z)$ ist analytisch in $z \in \mathscr{T}^{+}$,

(3) $\left\|\hat{T}_{\chi}(z)\right\| \leqq \sup _{\overline{V^{+}}}\left|e^{i(z, q)} \chi(q)\right|, z \in \overline{\mathscr{T}^{+}}$,

(4) $\hat{T}_{\chi}\left(z_{1}+z_{2}\right)=\hat{T}_{\chi}\left(z_{1}\right) \hat{T}\left(z_{2}\right)=\hat{T}\left(z_{1}\right) \hat{T}_{\chi}\left(z_{2}\right), z_{j} \in \overline{\mathscr{T}}^{+}$,

(5) $U(\Lambda) \hat{T}(z) U(\Lambda)^{-1}=\hat{T}(\Lambda z), \Lambda \in \mathscr{L}_{+}^{\dagger}, z \in \overline{\mathscr{T}}^{+}$.

Dabei wurde $\hat{T}_{1}(z)=\hat{T}(z)$ gesetzt, und $\Lambda \rightarrow U(\Lambda)$ ist die stetige unitäre Darstellung der orthochronen eigentlichen Lorentzgruppe $\mathscr{L}_{+}^{\uparrow}$, die gemäß den Wightman-Axiomen in $\mathfrak{G}$ existiert.

Beweis. Dieser Satz ist nur eine geringfügige Verallgemeinerung des Satzes 4 von [18], der die entsprechenden Aussagen für $\chi(q) \equiv 1$ macht. 
Zur weiteren Vorbereitung und zur Einführung unserer Bezeichnungen erinnern wir an einige Ergebnisse über Wightman-Funktionen [13].

$$
A_{1}\left(x_{1}\right) A_{2}\left(x_{2}\right) \Omega_{0} \equiv \Phi_{12}\left(x_{1}, x_{2}-x_{1}\right): \mathscr{S}\left(\mathbb{R}^{8}\right) \rightarrow \mathfrak{H}
$$

ist eine temperierte Distribution mit Werten im Hilbertraum $\mathfrak{S}$ der $\mathrm{Zu}$ stände. Die Fouriertransformierte $\tilde{\Phi}_{12}(p, q)$ hat ihren Träger in $p, q \in \operatorname{Tr} d E$, so daß $\Phi_{12}(x, \xi)$ Randwert im $\mathscr{S}^{\prime}$ einer in $(z, \zeta) \in \mathscr{T}_{2}^{+}$analytischen vektorwertigen Funktion $\Phi_{12}(z, \zeta)$ ist. Aus der Translationsinvarianz folgt für $z, z^{\prime}, \zeta \in \mathscr{T}^{+}$:

$$
\hat{T}\left(z^{\prime}\right) \Phi_{12}(z, \zeta)=\Phi_{12}\left(z^{\prime}+z, \zeta\right)
$$

und weiterhin, daß die Distribution

$$
\left(\Phi_{12}(x, f), \Phi_{12}(y, f)\right): \mathscr{S}\left(\mathbb{R}^{8}\right) \rightarrow \mathbb{C}
$$

für $f \in \mathscr{S}\left(\mathbb{R}^{4}\right)$ nur von $y-x$ abhängt. Die Positivität des Skalarproduktes in $\mathfrak{H}$ besagt dann gerade, daß $\left(\Phi_{12}(x, f), \Phi_{12}(y, f)\right) \equiv W(\breve{f}, y-x, f)$ in der Differenzvariablen eine temperierte Distribution vom positiven Typ ist. Folglich ist $W(\check{f}, \xi, f)$ Fouriertransformierte eines polynomialbeschränkten positiven Maßes mit Träger in $\operatorname{Tr} d E$ ([21], Satz von Bochner). Daraus folgt mit Lemma 1 von [5]:

$$
\left\|\Phi_{12}(z, \zeta)\right\| \leqq C\left|\zeta^{2}\right|^{N}\left(1+\frac{1}{d_{\zeta}}\right)^{M}\left(1+\frac{1}{d_{z}}\right)^{m}
$$

mit nichtnegativen ganzen Zahlen $N, M, m . d_{\zeta}$ bezeichnet den euklidischen Abstand des Punktes $\zeta \in \mathscr{T}^{+}$von $\mathbb{C}^{4} \backslash \mathscr{T}^{+}$, also

$$
d_{\zeta}=\frac{1}{\sqrt{2}}\left(\operatorname{Im} \zeta^{0}-|\operatorname{Im} \underline{\zeta}|\right) ; \quad \zeta=\left(\zeta^{0}, \underline{\zeta}\right) .
$$

Die zum Matrixelement

$$
\left(\Omega_{0}, A_{1}\left(x_{1}\right) A_{2}\left(x_{2}\right) A_{3}\left(x_{3}\right) A_{4}\left(x_{4}\right) \Omega_{0}\right)
$$

gehörige $W$-Funktion besitzt daher die Darstellung

$$
\mathfrak{W}_{1234}\left(z_{1}, z_{2}, z_{3}, z_{4}\right)=\left(\Phi_{12}^{*}\left(-\bar{z}_{2}, \bar{z}_{1}-\bar{z}_{2}\right), \Phi_{34}\left(z_{3}, z_{4}-z_{3}\right)\right) \text {. }
$$

Infolge der Translationsinvarianz haben wir

$$
\begin{gathered}
\mathfrak{W}_{1234}\left(z_{1}, z_{2}, z_{3}, z_{4}\right)=W_{1234}\left(z_{2}-z_{1}, z_{3}-z_{2}, z_{4}-z_{3}\right) \\
=\mathfrak{W}_{1234}\left(z_{1}-z_{2}+z, z, z_{3}-z_{2}+z, z_{4}-z_{2}+z\right)
\end{gathered}
$$


für beliebige $z \in \mathscr{T}^{+}, z_{j}-z_{j-1} \in \mathscr{T}^{+}, j=2,3,4$, also nach (II.4) und (II.2):

$$
\begin{aligned}
& \mathfrak{W}_{1234}\left(z_{1}, z_{2}, z_{3}, z_{4}\right)=\left(\Phi_{12}^{*}\left(-\bar{z}, \bar{z}_{1}-\bar{z}_{2}\right), \Phi_{34}\left(z_{3}-z_{2}+z, z_{4}-z_{3}\right)\right) \\
& \quad=\left(\Phi_{12}^{*}\left(-\bar{z}, \bar{z}_{1}-\bar{z}_{2}\right), \hat{T}\left(z_{3}-z_{2}\right) \Phi_{34}\left(z, z_{4}-z_{3}\right)\right) .
\end{aligned}
$$

Entsprechendes gilt für $\mathfrak{W}_{2134}, \mathfrak{B}_{1243}$ und $\mathfrak{M}_{2143}$. Damit sind die $W$-Funktionen als Matrixelemente des Translationsoperators zwischen geeigneten Zuständen dargestellt, die analytische Funktionen ihrer Variablen sind.

Die Lokalität der Theorie kann durch die Zustände $\Phi_{j k}$, folgendermaßen ausgedrückt werden.

Für $\left(x_{4}-x_{3}\right)^{2}<0$ gilt in $\mathscr{S}^{\prime}$

$$
\Phi_{34}\left(x_{3}, x_{4}-x_{3}\right)=\Phi_{43}\left(x_{4}, x_{3}-x_{4}\right) .
$$

Daraus folgt für beliebige $z \in \mathscr{T}^{+}, \xi^{2}<0$

$$
T\left(-\frac{1}{2} \xi\right) \Phi_{34}(z, \xi)=T\left(\frac{1}{2} \xi\right) \Phi_{43}(z,-\xi) .
$$

Es erweist sich für unsere Zwecke als vorteilhaft, folgende Koordinaten einzuführen:

$$
\begin{gathered}
\zeta_{1}=z_{2}-z_{1}, \quad \zeta_{3}=z_{4}-z_{3}, \quad \zeta=\frac{1}{2}\left(z_{3}+z_{4}-z_{1}-z_{2}\right), \\
\zeta_{2}=z_{3}-z_{2}=\zeta-\frac{1}{2}\left(\zeta_{1}+\zeta_{3}\right) .
\end{gathered}
$$

Die in diesen Variablen geschriebenen $W$-Funktionen $W_{1234}, W_{2134}$, $W_{1243}, W_{2143}$ bezeichnen wir mit $M_{11}, M_{21}, M_{12}, M_{22}$, d. h. ausführlich:

$$
\begin{aligned}
M_{11}\left(\zeta_{1}, \zeta, \zeta_{3}, z\right) & =\left(\Phi_{12}^{*}\left(-\bar{z},-\bar{\zeta}_{1}\right), \hat{T}\left(\zeta-\frac{1}{2}\left(\zeta_{1}+\zeta_{3}\right)\right) \Phi_{34}\left(z, \zeta_{3}\right)\right) \\
& =W_{1234}\left(\zeta_{1}, \zeta-\frac{1}{2}\left(\zeta_{1}+\zeta_{3}\right), \zeta_{3}\right), \\
M_{12}\left(\zeta_{1}, \zeta, \zeta_{3}, z\right) & =\left(\Phi_{12}^{*}\left(-\bar{z},-\bar{\zeta}_{1}\right), \hat{T}\left(\zeta-\frac{1}{2}\left(\zeta_{1}-\zeta_{3}\right)\right) \Phi_{43}\left(z,-\zeta_{3}\right)\right) \\
& =W_{1243}\left(\zeta_{1}, \zeta-\frac{1}{2}\left(\zeta_{1}-\zeta_{3}\right),-\zeta_{3}\right), \\
M_{22}\left(\zeta_{1}, \zeta, \zeta_{3}, z\right) & =\left(\Phi_{21}^{*}\left(-\bar{z}, \bar{\zeta}_{1}\right), \hat{T}\left(\zeta+\frac{1}{2}\left(\zeta_{1}+\zeta_{3}\right)\right) \Phi_{43}\left(z,-\zeta_{3}\right)\right) \\
& =W_{2143}\left(-\zeta_{1}, \zeta+\frac{1}{2}\left(\zeta_{1}+\zeta_{3}\right),-\zeta_{3}\right) \\
M_{21}\left(\zeta_{1}, \zeta, \zeta_{3}, z\right) & =\left(\Phi_{21}^{*}\left(-\bar{z}, \bar{\zeta}_{1}\right), \hat{T}\left(\zeta+\frac{1}{2}\left(\zeta_{1}-\zeta_{3}\right)\right) \Phi_{34}\left(z, \zeta_{3}\right)\right) \\
& =W_{2134}\left(-\zeta_{1}, \zeta+\frac{1}{2}\left(\zeta_{1}-\zeta_{3}\right), \zeta_{3}\right) .
\end{aligned}
$$

Die "Wightman-Funktionen" $M_{j k}$ sind analytisch in $\left(\zeta_{1}, \zeta, \zeta_{3}\right) \in \Omega_{j k}$ $:=\left\{\left(\zeta_{1}, \zeta, \zeta_{3}\right) \mid\left((-1)^{j+1} \zeta_{1}, \zeta+\frac{(-1)^{j}}{2} \zeta_{1}+\frac{(-1)^{k}}{2} \zeta_{3},(-1)^{k+1} \zeta_{3}\right) \in \mathscr{T}_{3}^{+}\right\}$ 
und $z \in \mathscr{T}^{+}$. Die Gebiete $\Omega_{j k}$ sind die den vier Permutationen (1234), (2134), (1243) und (2143) zugeordneten Vorwärtstuben. Für $j \neq j^{\prime}$ oder $k \neq k^{\prime}$ haben $\Omega_{j k}$ und $\Omega_{j^{\prime} k^{\prime}}$ keine Punkte gemeinsam. Die Lokalität und die $\mathscr{L}_{+}^{\uparrow}$-Invarianz der $W$-Funktionen garantieren die Existenz einer eindeutigen $\mathscr{L}_{+}(\mathbb{C})$-invarianten gemeinsamen analytischen Fortsetzung dieser vier Funktionen in die Vereinigung $\bigcup_{j, k=1,2} \Omega_{j k}^{\prime}$ der erweiterten "Tuben"

$$
\Omega_{j k}^{\prime}=\left\{\left(\zeta_{1}, \zeta, \zeta_{3}\right) \mid\left((-1)^{j+1} \zeta_{1}, \zeta+\frac{(-1)^{j}}{2} \zeta_{1}+\frac{(-1)^{k}}{2} \zeta_{3},(-1)^{k+1} \zeta_{3}\right) \in \mathscr{T}_{3}^{\prime}\right\}
$$

([13], p. 150).

Aus (II.6) und (II.3) gewinnen wir leicht Schranken für das Wachstum der Funktionen $M_{j k}$. Es ist z. B.

$$
\begin{aligned}
\left|M_{11}\left(\zeta_{1}, \zeta, \zeta_{3}, z\right)\right| & \leqq\left\|\Phi_{12}^{*}\left(-\bar{z},-\bar{\zeta}_{1}\right)\right\|\left\|\Phi_{34}\left(z, \zeta_{3}\right)\right\|\left\|\hat{T}\left(\zeta-\frac{1}{2}\left(\zeta_{1}+\zeta_{3}\right)\right)\right\| \\
& \leqq \hat{\psi}_{1}^{*}(z) \hat{\psi}_{1}(z) \psi_{1}^{*}\left(\zeta_{1}\right) \psi_{1}\left(\zeta_{3}\right)\left\|\hat{T}\left(\zeta-\frac{1}{2}\left(\zeta_{1}+\zeta_{3}\right)\right)\right\|
\end{aligned}
$$

Dabei sind $\hat{\psi}_{1}^{*}(z), \hat{\psi}_{1}(z)$ und $\psi_{1}^{*}\left(\zeta_{3}\right), \psi_{1}\left(\zeta_{3}\right)$ gemäß (II.3) definiert. Diese Abschätzung kann mit anderen Konstanten $C_{j}$ und nichtnegativen ganzen Zahlen $N_{j}, M_{j}, m_{j}$ auf die übrigen Funktionen $M_{j k}$ übertragen werden. Da wir bei den folgenden Überlegungen zunächst nicht explizit auf die " $z$-Abhängigkeit" der Zustände $\Phi_{j k}$ eingehen, schreiben wir abkürzend, z. B.

$$
\Phi_{34}\left(\zeta_{3}\right) \equiv \Phi_{34}\left(z, \zeta_{3}\right) \text { mit beliebigem } z \in \mathscr{T}^{+} \text {. }
$$

Die mit den Zuständen $\Phi_{j k}(z, \zeta)$ gemäß (II.6) gebildeten Matrixelemente $M_{j k}$ hängen nicht mehr von $z$ ab.

Durch

$$
\begin{aligned}
\Delta \rightarrow & M_{11}\left(\zeta_{1}, \zeta, \zeta_{3} ; \Delta\right) \\
& =\left(\Phi_{12}^{*}\left(-\bar{\zeta}_{1}\right), E(\Delta) \hat{T}\left(\zeta-\frac{1}{2}\left(\zeta_{1}+\zeta_{3}\right)\right) \Phi_{34}\left(\zeta_{3}\right)\right) \\
& =\left(\Phi_{12}^{*}\left(-\bar{\zeta}_{1}\right), \hat{T}_{\Delta}\left(\zeta-\frac{1}{2}\left(\zeta_{1}+\zeta_{3}\right)\right) \Phi_{34}\left(\zeta_{3}\right)\right)
\end{aligned}
$$

mit

$$
\hat{T}_{\Delta}(z)=\int_{\Delta} e^{i(z, p)} d E(p),
$$

gemäß Satz I.1 für Borelmengen $\Delta \subset \mathbb{R}^{4}$ wird ein beschränktes komplexwertiges $\mathrm{Ma}$ mit Träger in $\overline{V^{+}}$erklärt. Ist $\Delta \cap \overline{V^{+}}$beschränkt, so ist nach Satz I.1 $\hat{T}_{\Delta}(z)$ eine ganze analytische Funktion und daher

$$
M_{11}\left(\zeta_{1}, \zeta, \zeta_{3} ; \Delta\right)
$$


in $\left(\zeta_{1}, \zeta, \zeta_{3}\right) \in \mathscr{T}^{+} \times \mathbb{C}^{4} \times \mathscr{T}^{+}$analytisch (Satz von Hartogs, [11], p. 28). Infolge $E\left(\overline{V^{+}}\right)=1$ gilt in $\Omega_{11}$

$$
M_{11}\left(\zeta_{1}, \zeta, \zeta_{3}\right)=\int_{V^{+}} d M_{11}\left(\zeta_{1}, \zeta, \zeta_{3} ; p\right)
$$

Für die übrigen $M_{j k}$ gelten analoge Aussagen, insbesondere also in $\Omega_{j k}$

$$
M_{j k}\left(\zeta_{1}, \zeta, \zeta_{3}\right)=\int_{V^{+}} d M_{j k}\left(\zeta_{1}, \zeta, \zeta_{3} ; p\right)
$$

mit Maßen $d M_{j k}\left(\zeta_{1}, \zeta, \zeta_{3} ; p\right)$, die in

$$
\left((-1)^{j+1} \zeta_{1}, \zeta,(-1)^{k+1} \zeta_{3}\right) \in \mathscr{T}^{+} \times \mathbb{C}^{4} \times \mathscr{T}^{+}
$$

analytisch sind.

Sei $\varphi$ eine stetige Funktion, deren Träger in einer Kugel

$$
K_{\varepsilon^{\prime}}=\left\{\left(p^{0}, p^{1}, p^{2}, p^{3}\right) \mid\|p\|^{2}=\sum_{j=0}^{3}\left(p^{j}\right)^{2} \leqq \varepsilon^{\prime 2}\right\}
$$

liegt und die $\int \varphi(p) d^{4} p=1$ erfüllt.

Dann hat die Funktion $\varphi_{q}(p):=\varphi(q-p)$ ihren Träger in

$$
\begin{gathered}
\left\{p \mid\|p-q\| \leqq \varepsilon^{\prime}\right\} \subset\left(q^{\prime \prime}+\overline{V^{+}}\right) \cap\left(q^{\prime}-\overline{V^{+}}\right), \\
q^{\prime}:=q+\varepsilon, \quad q^{\prime \prime}:=q-\varepsilon, \quad \varepsilon:=\left(\sqrt{2} \varepsilon^{\prime}, \underline{0}\right) .
\end{gathered}
$$

Nach Satz II.1 und dem Satz von Hartogs ([12], p. 28) ist das Konvolutionsprodukt

$$
\begin{array}{r}
M_{11}\left(\zeta_{1}, \zeta, \zeta_{3} ; \varphi_{q}\right):=\int \varphi_{q}(p) d M_{11}\left(\zeta_{1}, \zeta, \zeta_{3} ; p_{3}\right) \\
=\left(\Phi_{12}^{*}\left(-\bar{\zeta}_{1}\right), \hat{T}_{\varphi_{q}}\left(\zeta-\frac{1}{2}\left(\zeta_{1}+\zeta_{3}\right)\right) \Phi_{34}\left(\zeta_{3}\right)\right)
\end{array}
$$

für feste $q$ eine in $\left(\zeta_{1}, \zeta, \zeta_{3}\right) \in \mathscr{T}^{+} \times \mathbb{C}^{4} \times \mathscr{T}^{+}$analytische Funktion mit $\operatorname{Tr}_{q} M_{11}\left(\zeta_{1}, \zeta, \zeta_{3} ; \varphi_{q}\right) \subset-\varepsilon+\overline{V^{+}} \equiv \overline{V_{\varepsilon}^{+}}$, die nach (II.7) durch

$$
\left|M_{11}\left(\zeta_{1}, \zeta, \zeta_{3} ; \varphi_{q}\right)\right| \leqq \hat{\psi}_{1}^{*}(z) \hat{\psi}_{1}(z) \psi_{1}^{*}\left(\zeta_{1}\right) \psi_{1}\left(\zeta_{3}\right)\left\|\hat{T}_{\varphi_{q}}\left(\zeta-\frac{1}{2}\left(\zeta_{1}+\zeta_{3}\right)\right)\right\|
$$

abgeschätzt wird.

Sei $\left(\zeta_{1}, \zeta, \zeta_{3}\right) \in \Omega_{11}$, also $y=\operatorname{Im}\left(\zeta-\frac{1}{2}\left(\zeta_{1}+\zeta_{3}\right)\right) \in V^{+}$, dann gilt

$$
\left\|\hat{T}_{\varphi_{q}}\left(\zeta-\frac{1}{2}\left(\zeta_{1}+\zeta_{3}\right)\right)\right\| \leqq \sup _{\overline{V_{\varepsilon}^{+}}}\left|\varphi_{q}(p)\right| e^{-\left(q^{\prime \prime}, y\right)} \equiv\|\varphi\| e^{-\left(q^{\prime \prime}, y\right)}
$$

da $\operatorname{Tr} \varphi_{q} \subset\left(q^{\prime \prime}+\overline{V^{+}}\right) \cap\left(q^{\prime}-\overline{V^{+}}\right)$. Ist hingegen nur

$$
\left(\zeta_{1}, \zeta, \zeta_{3}\right) \in \mathscr{T}^{+} \times \mathbb{C}^{4} \times \mathscr{T}^{+},
$$

so können wir mit derselben Begründung folgendermaßen abschätzen:

$$
\begin{aligned}
\left\|\hat{T}_{\varphi_{q}}\left(\zeta-\frac{1}{2}\left(\zeta_{1}+\zeta_{3}\right)\right)\right\| & \leqq K_{\zeta, \varphi_{q}} \cdot e^{\frac{1}{2}\left|\left(q^{\prime}, \operatorname{Im} \zeta_{1}\right)\right|+\frac{1}{2}\left|\left(q^{\prime}, \operatorname{Im} \zeta_{3}\right)\right|} \\
K_{\zeta, \varphi_{q}} & =\sup _{p}\left|e^{i(\zeta, p)} \varphi_{q}(p)\right| .
\end{aligned}
$$


Diese Überlegungen lassen sich auf die übrigen $M_{j k}$ übertragen; wir erhalten somit

$$
\begin{gathered}
\left|M_{j k}\left(\zeta_{1}, \zeta, \zeta_{3} ; \varphi_{q}\right)\right| \leqq \hat{\psi}_{j}^{*}(z) \hat{\psi}_{k}(z) \psi_{j}^{*}\left(\zeta_{1}\right) \psi_{k}\left(\zeta_{3}\right) \cdot A \\
A=\left\{\begin{array}{l}
\|\varphi\| \exp \left(-\left(q^{\prime \prime}, \operatorname{Im}\left(\zeta+\frac{(-1)^{j+1}}{2} \zeta_{1}+\frac{(-1)^{k+1}}{2} \zeta_{3}\right)\right)\right) \text { in } \Omega_{j k} \\
K_{\zeta, \varphi_{q}} \exp \left(\frac{1}{2} \sum_{j=1,3}\left|\left(q^{\prime}, \operatorname{Im} \zeta_{j}\right)\right|\right) \text { in }(-1)^{j+1} \mathscr{T}^{+} \times \mathbb{C}^{4} \times(-1)^{k+1} \mathscr{T}^{+} .
\end{array}\right.
\end{gathered}
$$

Für $y \in V^{+}$ist $\int_{\frac{V_{\varepsilon}^{+}}{}} e^{-\left(q^{\prime \prime}, y\right)} d^{4} q=16 \pi e^{2(\varepsilon, y)}(y, y)^{-2}$, d. h. nach (II.12), daß $\int\left|M_{j k}\left(\zeta_{1}, \zeta, \zeta_{3} ; \varphi_{q}\right)\right| d^{4} q$ für $\left(\zeta_{1}, \zeta, \zeta_{3}\right) \in \Omega_{j k}$ existiert und folglich

$$
\begin{aligned}
& \int M_{j k}\left(\zeta_{1}, \zeta, \zeta_{3} ; \varphi_{q}\right) d^{4} q=\int_{V^{+}}\left(\int \varphi_{q}(p) d^{4} q\right) d M_{j k}\left(\zeta_{1}, \zeta, \zeta_{3} ; p\right) \\
& =\int_{V^{+}} d M_{j k}\left(\zeta_{1}, \zeta, \zeta_{3} ; p\right)=M_{j k}\left(\zeta_{1}, \zeta, \zeta_{3}\right) .
\end{aligned}
$$

Diese Ergebnisse fassen wir zu einem Satz zusammen:

Satz II.2. Die Wightman-Funktionen $M_{j k}, j, k=1,2$ besitzen Integraldarstellungen

$$
M_{j k}\left(\zeta_{1}, \zeta, \zeta_{3}\right)=\int M_{j k}\left(\zeta_{1}, \zeta, \zeta_{3} ; \varphi_{q}\right) d^{4} q
$$

mit Funktionen $M_{j k}\left(\zeta_{1}, \zeta, \zeta_{3} ; \varphi_{q}\right)$, die in

$$
\left((-1)^{j+1} \zeta_{1}, \zeta,(-1)^{k+1} \zeta_{3}\right) \in \mathscr{T}^{+} \times \mathbb{C}^{4} \times \mathscr{T}^{+}
$$

analytisch sind und nicht stärker wachsen als

$$
\left|M_{j k}\left(\zeta_{1}, \zeta, \zeta_{3} ; \varphi_{q}\right)\right| \leqq K_{\zeta, \varphi_{q}} \hat{\psi}_{j}^{*}(z) \psi_{j}^{*}\left(\zeta_{1}\right) \hat{\psi}_{k}^{*}(z) \psi_{k}\left(\zeta_{3}\right) e^{\frac{1}{2} \sum_{j=1,3}\left|\left(q^{\prime}, \operatorname{Im} \zeta_{j}\right)\right|} .
$$

Dabei ist $\varphi$ eine beliebige Funktion mit der Eigenschaft (II.10) und $\operatorname{Tr} \varphi_{q} \subset\left(q^{\prime \prime}+\overline{V^{+}}\right) \cap\left(q^{\prime}-\overline{V^{+}}\right)$und

$$
\begin{aligned}
& K_{\zeta, \varphi_{q}}=\sup _{p}\left|e^{i(\zeta, p)} \varphi(q-p)\right| \\
& \psi_{j}(\zeta)=C_{j}|(\zeta, \zeta)|^{N_{j}}\left(1+\frac{1}{d_{\zeta}}\right)^{M_{j}}, \\
& \hat{\psi}_{k}(z)=\left(1+\frac{1}{d_{z}}\right)^{m_{k}} .
\end{aligned}
$$




\section{Wachstumseigenschaften analytischer Funktionen}

Es bezeichne $\mathscr{T}_{n}^{+}$die Vorwärtstube in $n$-Vierervektoren. Sind $f^{+}$bzw. $f^{-}$in $\mathscr{T}_{n}^{+}$bzw. $\mathscr{T}_{n}^{-}=-\mathscr{T}_{n}^{+}$analytische Funktionen, deren Randwerte als Distributionen existieren und auf einem Gebiet $G \subset \mathbb{R}^{4}$ als Distributionen übereinstimmen, so liefert die Anwendung des "Edge of the Wedge"-Theorems $([17]$, p. 74,94$)$ eine gemeinsame analytische Fortsetzung $f$ von $f^{+}$und $f^{-}$nach $\mathscr{T}_{n}^{+} \cup \mathscr{T}_{n}^{-} \cup \tilde{G}$. Dabei ist $\tilde{G}$ eine offene Umgebung im $\mathbb{C}^{4 \cdot n}$ von $G$. Da $\mathscr{T}_{n}^{+} \cup \mathscr{T}_{n}^{-} \cup \tilde{G}$ im allgemeinen kein Holomorphiegebiet ist, taucht sogleich die Frage nach der Holomorphiehülle $\mathscr{H}\left(\mathscr{T}_{n}^{+} \cup \mathscr{T}_{n}^{-} \cup \tilde{G}\right)$ von $\mathscr{T}_{n}^{+} \cup \mathscr{T}_{n}^{-} \cup \tilde{G}$ auf. Bei der Anwendung dieser Situation auf Feldtheorien kennt man meistens die Wachstumseigenschaften der Funktion $f$ in $\mathscr{T}_{n}^{+} \cup \mathscr{T}_{n}^{-} \cup \tilde{G}$ und ist aus verschiedenen Gründen (Beweis von Dispersionsrelationen, Berechnung von Holomorphiehüllen) auch an einer Kenntnis des Wachstums der analytischen

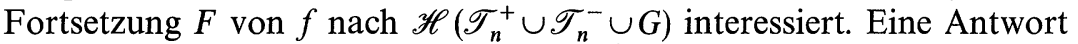
auf dieses Problem ist für spezielle Situationen bekannt:

In [15] ist ein Theorem von Bros bewiesen, welches besagt: Ist $f$ in $\mathscr{T}^{+} \cup \mathscr{T}^{-} \cup \tilde{\mathscr{R}}$ analytisch und gilt dort $|f(z)|<C e^{\alpha \mid \operatorname{Im} z} \mid, \alpha>0$, so gilt für die analytische Fortsetzung $F$ in $\mathscr{H}\left(\mathscr{T}^{+} \cup \mathscr{T}^{-} \cup \tilde{\mathscr{R}}\right):|F(z)| \leqq C e^{\alpha \mid \operatorname{Im} z} \mid$ Dabei ist $\mathscr{R}$ von recht spezieller Gestalt [15].

Eine zweite Aussage dieser Art haben wir in [7].

Lemma A2.1. Ist $T_{n}^{+}$die $n$-Punkt-Vorwärtstube des zweidimensionalen komplexen Minkowskiraumes und ist $F$ eine in der erweiterten Tube $T_{n}^{\prime}$ analytische Funktion, die in $T_{n}^{+} \cup T_{n}^{-} \cup I_{n}\left(I_{n}=T_{n}^{\prime} \cap \mathbb{R}^{2 \cdot n}\right)$

$$
\left|F\left(z_{1}, \ldots, z_{n}\right)\right|<C e^{\alpha \sum_{j=1}^{n}\left|\operatorname{Im} z_{j}^{0}\right|}, \quad z_{j}=\left(z_{j}^{0}, z_{j}^{1}\right) \in \mathbb{C}^{2}
$$

erfüllt, so gilt in $T_{n}^{\prime}$ :

$$
\left|F\left(z_{1}, \ldots, z_{n}\right)\right| \leqq C e^{\alpha \sum_{j=1}^{n} \max \left\{\left|\operatorname{Im} z_{j}^{0}\right|,\left|\operatorname{Im} z_{j}^{1}\right|\right\}}
$$

In der oben angegebenen allgemeinen Form ist das Problem der Kontrolle des Wachstums in der Holomorphiehülle von $\mathscr{T}_{n}^{+} \cup \mathscr{T}_{n}^{-} \cup \tilde{G}$ bisher nicht gelöst. Eine Lösung desselben würde beträchtliche Fortschritte in der Erweiterung des Holomorphiegebietes der $n$-PunktFunktionen und insbesondere der Streuamplitude bringen.

Mit Hilfe solcher Aussagen könnten auch leicht Schranken für das Wachstum der gemeinsamen analytischen Fortsetzung der $M_{j k}$ von Satz II.2 angegeben werden. Doch der Fall der $M_{j k}$ ist spezieller, kann er doch, wie sich zeigen wird, auf die Kontrolle des Wachstums der 
analytischen Fortsetzung „modifizierter“ Zwei-Punkt-Funktionen zurückgeführt werden, und das gelingt am einfachsten mit Hilfe der JostLehmann-Dyson-Darstellung [19], könnte aber auch mit Hilfe von Theorem II aus [15] und einem Theorem von Bros bewiesen werden.

Für seine klärenden Worte über diese Problematik und die Konsequenzen der Kontrolle der Wachstumseigenschaften exponentiell beschränkter analytischer Funktionen bei Edge-of-the-Wedge-Problemen möchte ich Prof. Glaser herzlich danken.

Dieser Abschnitt soll Hilfsmittel zur Kontrolle des Wachstums der analytischen Fortsetzung der $M_{j k}\left(\zeta_{1}, \zeta, \zeta_{3}, \varphi_{q}\right)$, deren Existenz im vierten Abschnitt nachgewiesen wird, bereitstellen. Dazu dient

Satz III.1. Ist eine Funktion $F$ in der erweiterten Tube $\mathscr{T}^{\prime}$ analytisch und in $\mathscr{T}^{+} \cup \mathscr{T}^{-} \cup \mathscr{I}$ durch

$$
|F(z)| \leqq C_{0} e^{|(k, \operatorname{Im} z)|}(1+\|z\|)^{n}\left(1+\frac{1}{d_{z}}\right)^{m}
$$

beschränkt ( $k \in \bar{V}^{+}$fest; $n, m$ nichtnegative ganze Zahlen und $d_{z}=$ euklidischer Abstand des Punktes z vom Rand von $\left.\mathscr{T}^{+} \cup \mathscr{T}^{-} \cup \mathscr{I}\right)$, dann gilt für alle $z \in \mathscr{T}^{\prime}$

$$
|F(z)| \leqq C_{1} e^{\max \left\{|(k, \operatorname{Im} z)|,\left[(k, \operatorname{Im} z)^{2}-k^{2}(\operatorname{Im} z)^{2}\right]^{1 / 2}\right\}}(1+\|z\|)^{N}\left(1+\frac{1}{d_{z}}\right)^{M}
$$

mit ganzen Zahlen $N=N(n, m) \geqq 0, M=M(n, m) \geqq 0$ und einer Konstanten $C_{1} \geqq C_{0}$, die unabhängig von $k \in \bar{V}^{+1}$ ist.

Beweis. Setze

$$
G_{ \pm}(z):=e^{ \pm i(k, z)} F_{ \pm}(z) \quad \text { für } \quad z \in \mathscr{T}^{ \pm}
$$

und $F_{ \pm}:=F \mid \mathscr{T}^{ \pm}$; dann gilt für die in $\mathscr{T}^{+}$bzw. $\mathscr{T}^{-}$analytischen Funktionen $G_{+}$bzw. $G_{-}$

$$
\left|G_{ \pm}(z)\right| \leqq C_{0}(1+\|z\|)^{n}\left(1+\frac{1}{d_{z}}\right)^{m}, z \in \mathscr{T}^{ \pm} .
$$

Nach dem Laplace-Transformierten-Satz (z.B. [19]) ist daher $G_{+}$bzw $G_{-}$ Laplace-Transformierte einer temperierten Distribution $\tilde{G}_{+}$bzw. $\tilde{G}_{-}^{-}$ mit Träger in $\bar{V}^{+}$bzw. $\bar{V}^{-}$, und die Randwerte

$$
g_{ \pm}(x):=\lim _{\substack{y \rightarrow 0 \\ y \in V^{+}}} G_{ \pm}(x \pm i y)
$$

in $\mathscr{S}^{\prime}\left(\mathbb{R}^{4}\right)$ von $G_{ \pm}(z)$ sind gerade die Fouriertransformierten dieser Distributionen $\tilde{G}_{ \pm}$. 
Daraus ergibt sich: $F_{+}(z)$ und $F_{-}(z)$ sind Laplace-Transformierte von temperierten Distributionen $\tilde{F}_{+}$und $\tilde{F}_{-}$, nämlich von

$$
\tilde{F}_{ \pm}(p):=\tilde{G}_{ \pm}( \pm k+p)
$$

die ihren Träger in $-k+\overline{V^{+}}$und $k-\overline{V^{+}}$haben. Der Träger

$$
\left(-k+\overline{V^{+}}\right) \cup\left(k+\overline{V^{-}}\right)
$$

von

$$
\tilde{H}(p):=\tilde{F}_{+}(p)-\tilde{F}_{-}(p)
$$

ist regulär im Sinne von Schwartz [21]. Demnach ist die Aufspaltung von $\tilde{H}(p)$ in die Differenz zweier temperierter Distributionen mit Träger in $-k+\overline{V^{+}}$bzw. $k-\overline{V^{+}}$auf verschiedene Weisen möglich; die Nichteindeutigkeit besteht in einer temperierten Distribution $\tilde{g}$ mit Träger in

$$
D(k):=\left(-k+\overline{V^{+}}\right) \cap\left(k-\overline{V^{+}}\right),
$$

d. h. gilt auch

$$
\tilde{H}(p)=\tilde{F}_{1}(p)-\tilde{F}_{2}(p), \quad \operatorname{Tr} \tilde{F}_{1} \subset-k+\overline{V^{+}}, \operatorname{Tr} \tilde{F}_{2} \subset k-\overline{V^{+}}
$$

so ist

$$
\tilde{F}_{1}(p)=\tilde{F}_{+}(p)+\tilde{g}(p)
$$

und

$$
\tilde{F}_{2}(p)=\tilde{F}_{-}(p)+\tilde{g}(p)
$$

mit einem $\tilde{g} \in \mathscr{S}^{\prime}\left(\mathbb{R}^{4}\right)$ und $\operatorname{Tr} \tilde{g} \subset D(k)$.

Nach Voraussetzung ist $F(z)$ in $\mathscr{I}=\left\{x \in \mathbb{R}^{4} \mid x^{2}<0\right\}$ holomorph, folglich $F_{+}(x)=F_{-}(x)$ für $x^{2}<0$, d. h. die Fouriertransformierte $H(x) \in \mathscr{S}^{\prime}\left(\mathbb{R}^{4}\right)$ von $\tilde{H}(p)$ verschwindet als Distribution für $x^{2}<0$. Damit erfüllt $\tilde{H}(p)$ die Voraussetzungen für eine Jost-Lehmann-Dyson-Darstellung zum Koinzidenzgebiet

$$
\mathscr{R}_{k}=\mathbb{R}^{4} \backslash\left(-k+\overline{V^{+}}\right) \cup\left(k-\overline{V^{+}}\right),
$$

und es resultiert die Darstellung

$$
\tilde{H}(p)=\int_{\mathcal{N}\left(\mathscr{R}_{k}\right)} d^{4} q d \varkappa^{2} \varepsilon\left(p^{0}-q^{0}\right) \delta\left((p-q)^{2}-\varkappa^{2}\right) h\left(q, \varkappa^{2}\right) .
$$

Dabei ist $h\left(q, \varkappa^{2}\right)$ eine temperierte Distribution, die ihren Träger in der Menge $\mathscr{N}\left(\mathscr{R}_{k}\right)$ der Parameter $\left(q, \varkappa^{2}\right) \in \mathbb{R}^{5}$ hat, die ein für $\mathscr{R}_{k}$ zulässiges Hyperboloid definieren [19]. Die Ordnung der temperierten Distribution $h\left(q, x^{2}\right)$ ist durch die Ordnung der Distributionen $\tilde{G}_{ \pm}, d$. h. durch $n$ und $m$ bestimmt und damit unabhängig von $k \in \overline{V^{+}}$. Nun ist für unseren 
Fall $\mathscr{N}\left(\mathscr{R}_{k}\right)=D(k) \times \mathbb{R}^{+}$, daher

$$
\tilde{H}(p)=\int_{D(k)} d^{4} q \tilde{f}(p-q, q)
$$

mit einer Distribution

$$
\tilde{f}(p, q)=\int_{0}^{\infty} d \varkappa^{2} \varepsilon\left(p^{0}\right) \delta\left(p^{2}-\varkappa^{2}\right) h\left(q, \varkappa^{2}\right),
$$

deren Träger in der Menge $\left\{(p, q) \mid p^{2} \geqq 0, q \in D(k)\right\}$ enthalten ist und deren partielle Fouriertransformierte $f(x, q)$ bezüglich $p$ für $x^{2}<0$ verschwindet.

Diese Trägereigenschaften von $\tilde{f}(p, q)$ und $f(x, q)$ gestatten eine JostLehmann-Dyson-Darstellung von $f(x, \mathrm{q})$ im $x$-Raum zum Koinzidenzgebiet $\mathscr{I}$ :

$$
f(x, q)=\int_{\mathscr{W}(\mathscr{G})} d^{4} u d \sigma^{2} \varepsilon\left(x^{0}-u^{0}\right) \delta\left((x-u)^{2}-\sigma^{2}\right) \psi\left(u, \sigma^{2} ; q\right)
$$

$\left(\psi=\right.$ getrennt stetiges bilineares Funktional auf $\mathscr{S}\left(\mathbb{R}_{u, \sigma}^{5}\right) \times \mathscr{S}\left(\mathbb{R}_{q}^{4}\right)$ mit $\operatorname{Tr} \psi \subset \mathscr{N}(\mathscr{I}) \times D(k))$.

Bis auf eine temperierte Distribution mit Träger in $p=0$ kann $\tilde{f}(p, q)$ eindeutig in

$$
\tilde{f}(p, q)=\tilde{f_{+}}(p, q)-\tilde{f_{-}}(p, q), \quad \operatorname{Tr} \tilde{f_{ \pm}} \subset\left\{(p, q) \mid p \in \overline{V^{ \pm}}, q \in D(k)\right\}
$$

aufgespalten werden. Deren Laplace-Transformierte $\hat{f}_{ \pm}$sind dann in $(z, \zeta) \in \mathscr{T}^{ \pm} \times \mathbb{C}^{4}$ analytische Funktionen mit bekanntem Wachstum (Laplace-Transformierten-Satz; Satz von Paley-Wiener-Schwartz):

$$
\left|\hat{f}_{ \pm}(z, \zeta)\right| \leqq C_{ \pm}(1+\|z\|)^{n_{ \pm}}\left(1+\frac{1}{d_{z}}\right)^{m_{ \pm}}(1+\|\zeta\|)^{n_{ \pm}^{\prime}} \sup _{q \in D(k)}\left\{\left|e^{i(q, \zeta)}\right|\right\}
$$

Die Ordnung der Distribution $\psi\left(u, \sigma^{2} ; q\right)$ in (III.4) bestimmt eine untere Schranke für $n \in \mathbb{N}$ so, daß durch

$$
\begin{aligned}
& \hat{f}(z, \zeta):=\int_{D(k)} d^{4} q e^{i(q, \zeta)} \frac{i}{2 \pi} \int_{\mathcal{N}(\mathscr{G})} d^{4} u d \sigma^{2} \psi\left(u, \sigma^{2} ; q\right) \\
& \cdot\left[(z-u)^{2}-\sigma^{2}\right]^{-1}\left[(z-u)^{2}+\sigma_{0}^{2}\right]^{n}\left[\sigma^{2}+\sigma_{0}^{2}\right]^{-n}, \quad \sigma_{0}^{2}>0
\end{aligned}
$$

eine im direkten Produkt der Jost-Lehmann-Dyson-Hülle von $\mathscr{T}^{+} \cup \mathscr{T}^{-} \cup \mathscr{I}$ und dem $\mathbb{C}^{4}$, d. h. in $\mathscr{T}^{\prime} \times \mathbb{C}^{4}$ analytische Funktion erklärt wird. Infolge der Nichteindeutigkeit der Aufspaltung $\tilde{f}=\tilde{f}_{+}-\tilde{f}_{-}$ist zunächst nur

$$
\hat{f}(z, \zeta)-\hat{f}_{ \pm}(z, \zeta)=\sum_{|v| \leqq N<\infty} a_{v}(\zeta) z^{v}, \quad(z, \zeta) \in \mathscr{T}^{ \pm} \times \mathbb{C}^{4}
$$


mit ganzen Funktionen $a_{v}(\zeta)$, die Laplace-Transformierte von Distributionen $\tilde{a}_{v}(q)$ mit Träger $D(k)$ sind [19]. Eine einfache Redefinition von $\hat{f}(z, \zeta)$, die aber die Wachstumseigenschaften nicht ändert, macht $\hat{f}(z, \zeta)$ zur gemeinsamen analytischen Fortsetzung von $\hat{f}_{ \pm}(z, \zeta)$ nach $\mathscr{T}^{\prime} \times \mathbb{C}^{4}$.

Der Aufspaltung von $\tilde{f}$ in $\tilde{f}_{ \pm}$entspricht die Aufspaltung

$$
\tilde{H}(p)=\tilde{H}_{+}(p)-\tilde{H}_{-}(p):=\int_{D(k)} d^{4} q \tilde{f}_{+}(p-q, q)-\int_{D(k)} d^{4} q \tilde{f}_{-}(p-q, q)
$$

von $\tilde{H}$ in $\tilde{H}_{ \pm}$mit $\operatorname{Tr} \tilde{H}_{ \pm} \subset \mp k \pm \overline{V^{+}}$. Die gemeinsame analytische Fortsetzung der Laplace-Transformierten

$$
\hat{H}_{ \pm}(z)=\int d^{4} p e^{i(p, z)} \tilde{H}_{ \pm}(p)=\hat{f}_{ \pm}(z, z)
$$

von $\tilde{H}_{ \pm}$erhalten wir daher in

$$
\begin{aligned}
\hat{H}(z) & =\hat{f}(z, z) \\
& =\int_{D(k)} d^{4} q e^{i(q, z)} \frac{i}{2 \pi} \int_{\mathscr{H}(\mathscr{I})} d^{4} u d \sigma^{2} \psi\left(u, \sigma^{2} ; q\right)\left[(z-u)^{2}-\sigma^{2}\right]^{-1} \\
& {\left[(z-u)^{2}+\sigma_{0}^{2}\right]^{n}\left[\sigma^{2}+\sigma_{0}^{2}\right]^{-n} . }
\end{aligned}
$$

Aus dieser Darstellung folgt: Es gibt natürliche Zahlen $N_{1}, M_{1}$ und eine Konstante $C_{1}$, so daß gilt:

$$
|\hat{H}(z)| \leqq C_{1}(1+\|z\|)^{N_{1}}\left(1+\frac{1}{d_{z}}\right)^{M_{1}} \sup _{p \in D(k)}\left\{\left|e^{i(p, z)}\right|\right\}
$$

für alle $z \in \mathscr{T}^{\prime}$.

Nach (III.1) und (III.2) unterscheidet sich die analytische Fortsetzung $\hat{H}$ von $\hat{H}_{+}$und $\hat{H}_{-}$von der gegebenen Funktion $F(z)$ höchstens um die Laplace-Transformierte einer Distribution $\tilde{g}(p)$ mit Träger in $D(k)$ :

$$
F(z)=\hat{H}(z)+\int d^{4} p e^{i(p, z)} \tilde{g}(p) .
$$

Aus der Ungleichung (III.7) und

$$
\left|\int d^{4} p e^{i(p, z)} \tilde{g}(p)\right| \leqq C_{2}(1+\|z\|)^{N_{2}} \sup _{p \in D(k)}\left\{\left|e^{i(p, z)}\right|\right\}
$$

folgt die Behauptung, denn es ist

$$
\sup _{p \in D(k)}\{-(\eta, p)\}=\max \left\{|(\eta, k)|,\left[(\eta, k)^{2}-\eta^{2} k^{2}\right]^{1 / 2}\right\} .
$$

Die Aussage von Satz III.1 kann unter einem etwas anderen Gesichtspunkt folgendermaßen formuliert werden; diese Form entspricht dann der für die Anwendung auf Feld-Theorien typischen Situation und liegt auch unserer Anwendung zugrunde. 
Satz III.1'. Es seien $F_{1}(x)$ und $F_{2}(x)$ temperierte Distributionen, die für $x^{2}<0$ als Distributionen übereinstimmen. Die Träger ihrer Fouriertransformierten $\tilde{F}_{1}(p) b z w . \tilde{F}_{2}(p)$ seien in $-k+\overline{V^{+}}$bzw. $k-\overline{V^{+}}$enthalten $\left(k \in \bar{V}^{+}\right.$fest). Dann sind $F_{1}(x)$ und $F_{2}(x)$ verschiedene Randwerte einer in der erweiterten Tube $\mathscr{T}^{\prime}$ analytischen Funktion $\hat{F}(z)$, die nicht stärker wächst als

$$
|\hat{F}(z)| \leqq C_{0} \cdot e^{\max \left\{|(k, \operatorname{Im} z)|,\left[(k, \operatorname{Im} z)^{2}-k^{2}(\operatorname{Im} z)^{2}\right]^{1 / 2}\right\}}(1+\|z\|)^{N}\left(1+\frac{1}{d_{z}}\right)^{M} .
$$

Die ganzen Zahlen $N, M \geqq 0$ sind durch die Ordnung der Distribution $\tilde{F}_{1}(p)-\tilde{F}_{2}(p)$ bestimmt.

Zum Beweis kann man wie oben direkt die Jost-Lehmann-DysonDarstellung anwenden, oder man benutzt den Laplace-TransformiertenSatz und das Edge-of-the-Wedge Theorem [17], um diesen Satz auf den obigen zurückzuführen.

\section{Gemeinsame analytische Fortsetzung von Wightman-Funktionen, die einen doppelten Kommutator erzeugen}

Zunächst wird die Existenz einer gemeinsamen analytischen Fortsetzung $M\left(\zeta_{1}, \zeta, \zeta_{3} ; \varphi_{q}\right)$ der in Abschnitt II eingeführten Funktionen $M_{j k}\left(\zeta_{1}, \zeta, \zeta_{3} ; \varphi_{q}\right)$ nach $\mathscr{T}^{\prime} \times \mathbb{C}^{4} \times \mathscr{T}^{\prime}$ bewiesen. Mit Hilfe der Ergebnisse von Abschnitt III kann das Wachstum dieser Funktionen in $\mathscr{T}^{\prime} \times \mathbb{C}^{4} \times \mathscr{T}^{\prime}$ kontrolliert werden (Satz IV.1). Damit ist es möglich, eine analytische Fortsetzung $M\left(\zeta_{1}, \zeta, \zeta_{3}\right)$ der $M_{j k}\left(\zeta_{1}, \zeta, \zeta_{3}\right)$, die zwei Kommutatoren erzeugen, als Integral über $M\left(\zeta_{1}, \zeta, \zeta_{3} ; \varphi_{q}\right)$ anzugeben (Satz IV.2). Abschließend diskutieren wir das auf diese Weise gewonnene Holomorphiegebiet der Funktion $M\left(\zeta_{1}, \zeta, \zeta_{3}\right)$.

Satz IV.1. Die in Satz II.2 eingeführten Funktionen $M_{j k}\left(\zeta_{1}, \zeta, \zeta_{3} ; \varphi_{q}\right)$ besitzen für jedes feste $\varphi$ mit der Eigenschaft (II.10) und jedes $q \in \mathbb{R}^{4}$ eine eindeutige gemeinsame analytische Fortsetzung $M\left(\zeta_{1}, \zeta, \zeta_{3} ; \varphi_{q}\right)$ nach $\mathscr{T}^{\prime} \times \mathbb{C}^{4} \times \mathscr{T}^{\prime}$. Es gilt

$$
\begin{gathered}
\operatorname{Tr}_{q} M\left(\zeta_{1}, \zeta, \zeta_{3} ; \varphi_{q}\right) \subset \overline{V_{\varepsilon}^{+}}=-\varepsilon+\overline{V^{+}} \\
\left|M\left(\zeta_{1}, \zeta, \zeta_{3} ; \varphi_{q}\right)\right| \leqq \hat{\psi}(z) K_{\zeta, \varphi_{q}} \psi^{*}\left(\zeta_{1}\right) \psi\left(\zeta_{3}\right) e^{\chi\left(q^{\prime}, \operatorname{Im} \zeta_{1}, \operatorname{Im} \zeta_{3}\right)} \\
\chi\left(q^{\prime}, \eta_{1}, \eta_{3}\right):=\frac{1}{2} \sum_{j=1,3} \max \left\{\left|\left(q^{\prime}, \eta_{j}\right)\right|,\left[\left(q^{\prime}, \eta_{j}\right)^{2}-q^{\prime 2} \eta_{j}^{2}\right]^{\frac{1}{2}}\right\} \\
\psi(\zeta)=C|(\zeta, \zeta)|^{N}\left(1+\frac{1}{d_{\zeta}}\right)^{M}
\end{gathered}
$$


mit gewissen ganzen Zahlen $N, M \geqq 0,0<C<\infty$, die unabhängig von $q$ sind. $\hat{\psi}(z)$ ist so bestimmt, daß

$$
\hat{\psi}_{j}^{*}(z) \hat{\psi}_{k}(z)=\left(1+\frac{1}{d_{z}}\right)^{m_{j}^{*}+m_{k}} \leqq\left(1+\frac{1}{d_{z}}\right)^{m} \equiv \hat{\psi}(z)
$$

für $j, k=1,2$ gilt.

$K_{\zeta, \varphi_{q}}, \varepsilon, q^{\prime}$ sind wie in Satz II.2 erklärt.

Beweis. 1. Es bezeichne

$$
M_{11}\left(\xi_{1}+i 0, \zeta, \zeta_{3} ; \varphi_{q}\right)=\lim _{V+\ni \eta_{1} \rightarrow 0} M_{11}\left(\xi_{1}+i \eta_{1}, \zeta, \zeta_{3} ; \varphi_{q}\right)
$$

den Randwert in $\mathscr{S}^{\prime}\left(\mathbb{R}^{4}\right)$, der in $\left(\zeta_{1}, \zeta, \zeta_{3}\right) \in \mathscr{T}^{+} \times \mathbb{C}^{4} \times \mathscr{T}^{+}$analytischen Funktion. Die Abschätzung für $M_{11}\left(\zeta_{1}, \zeta, \zeta_{3} ; \varphi_{q}\right)$ in Satz II.2 garantiert die Existenz dieses Randwertes in $\mathscr{S}^{\prime}([19]$, p. 230 ; [4]) und die Analytizität von $M_{11}\left(\xi_{1}+i 0, \zeta, \zeta_{3} ; \varphi_{q}\right)$ in $\left(\zeta, \zeta_{3}\right) \in \mathbb{C}^{4} \times \mathscr{T}^{+}$.

Entsprechend sei

$$
M_{21}\left(\xi_{1}-i 0, \zeta, \zeta_{3} ; \varphi_{q}\right):=\lim _{V^{+} \ni \eta_{1} \rightarrow 0} M_{21}\left(\xi_{1}-i \eta_{1}, \zeta, \zeta_{3} ; \varphi_{q}\right) .
$$

Mit Hilfe von Gl. (II.5) liefert die Lokalität der Theorie, daß in $\mathscr{S}^{\prime}(\mathscr{I})$ gilt $\left(\left(\zeta, \zeta_{3}\right) \in \mathbb{C}^{4} \times \mathscr{T}^{+}\right.$fest $)$:

$$
M_{11}\left(\xi_{1}+i 0, \zeta, \zeta_{3} ; \varphi_{q}\right)=M_{21}\left(\xi_{1}-i 0, \zeta, \zeta_{3} ; \varphi_{q}\right) .
$$

Das "Edge of the Wedge"-Theorem ([17], p. 74ff.) erlaubt, für feste $\left(\zeta, \zeta_{3}\right) \in \mathbb{C}^{4} \times \mathscr{T}^{+}$auf die Existenz einer gemeinsamen analytischen Fortsetzung $M_{1}\left(\zeta_{1}, \zeta, \zeta_{3} ; \varphi_{q}\right)$ in $\zeta_{1}$ nach $\zeta_{1} \in \mathscr{T}^{+} \cup \mathscr{T}^{-} \cup \mathscr{I}$ zu schließen, die dann nach dem Satz von Hartogs sogar in $\left(\zeta_{1}, \zeta, \zeta_{3}\right) \in\left(\mathscr{T}^{+} \cup \mathscr{T}^{-} \cup \mathscr{I}\right)$ $\times \mathbb{C}^{4} \times \mathscr{T}^{+}$analytisch ist und folglich nach dem Theorem von Glaser und Streater ([13], p. 92; [16]) nach $\mathscr{T}^{\prime} \times \mathbb{C}^{4} \times \mathscr{T}^{+}$analytisch fortgesetzt werden kann.

In $\zeta_{1} \in \mathscr{T}^{+} \cup \mathscr{T}^{-} \cup \mathscr{I},\left(\zeta, \zeta_{3}\right) \in \mathbb{C}^{4} \times \mathscr{T}^{+}$ist das Wachstum von $M_{1}$ durch Satz II.2 bestimmt:

$$
\left|M_{1}\left(\zeta_{1}, \zeta, \zeta_{3} ; \varphi_{q}\right)\right| \leqq K_{\zeta, \varphi_{q}} \hat{\psi}(z) \hat{\psi}^{*}\left(\zeta_{1}\right) \psi_{1}\left(\zeta_{3}\right) e^{\frac{1}{2} \sum_{j=1,3}\left|\left(q^{\prime}, \operatorname{Im} \zeta_{j}\right)\right|}
$$

wo

$$
\begin{gathered}
\hat{\psi}^{*}(\zeta)=\hat{C}^{*}|(\zeta, \zeta)|^{N^{*}}\left(1+\frac{1}{d_{\zeta}}\right)^{\hat{M}^{*}} \\
\hat{C}^{*}=\max \left\{C_{1}^{*}, C_{2}^{*}\right\}, \quad N^{*}=\max \left\{N_{1}^{*}, N_{2}^{*}\right\}, \quad \hat{M}^{*}=\max \left\{M_{1}^{*}, M_{2}^{*}\right\}
\end{gathered}
$$

unabhängig von $\varphi$ und $q$ sind.

Daraus folgt nach Satz (III.1) für $\zeta_{1} \in \mathscr{T}^{\prime},\left(\zeta, \zeta_{3}\right) \in \mathbb{C}^{4} \times \mathscr{T}^{+}$

$$
\begin{array}{r}
\left|M_{1}\left(\zeta_{1}, \zeta, \zeta_{3} ; \varphi_{q}\right)\right| \leqq \hat{\psi}(z) K_{\zeta, \varphi_{j}} \psi_{1}\left(\zeta_{3}\right) e^{\frac{1}{2}\left|\left(q^{\prime}, \operatorname{Im} \zeta_{3}\right)\right|} \\
\cdot \psi^{*}\left(\zeta_{1}\right) e^{\frac{1}{2} \max \left\{\left|\left(q^{\prime}, \operatorname{Im} \zeta_{1}\right)\right|,\left[\left(q^{\prime}, \operatorname{Im} \zeta_{1}\right)^{2}-q^{\prime 2}\left(\operatorname{Im} \zeta_{1}\right)^{2}\right]^{1 / 2}\right\}}
\end{array}
$$


mit

$$
\psi^{*}\left(\zeta_{1}\right)=C^{*}\left|\left(\zeta_{1}, \zeta_{1}\right)\right|^{N^{*}}\left(1+\frac{1}{d_{\zeta_{1}}}\right)^{M^{*}} ; M^{*}=M^{*}\left(\hat{M}^{*}, N^{*}\right) \in \mathbb{N}
$$

und $C^{*}=C^{*}\left(\hat{C}^{*}, N^{*}, \hat{M}^{*}\right)$ sind unabhängig von $\varphi$ und $q$ nach Satz III.1 bestimmt.

In gleicher Weise erhalten wir eine analytische Fortsetzung

$$
M_{2}\left(\zeta_{1}, \zeta, \zeta_{3} ; \varphi_{q}\right)
$$

von $M_{12}$ und $M_{22}$ nach $\mathscr{T}^{\prime} \times \mathbb{C}^{4} \times \mathscr{T}^{-}$mit

$$
\begin{gathered}
\left|M_{2}\left(\zeta_{1}, \zeta, \zeta_{3} ; \varphi_{q}\right)\right| \leqq K_{\zeta, \varphi_{q}} \hat{\psi}(z) \psi_{2}\left(\zeta_{3}\right) e^{\frac{1}{2}\left|\left(q^{\prime}, \operatorname{Im} \zeta_{3}\right)\right|} \\
\cdot \psi^{*}\left(\zeta_{1}\right) e^{\frac{1}{2} \max \left\{\left|\left(q^{\prime}, \operatorname{Im} \zeta_{1}\right)\right|,\left[\left(q^{\prime}, \operatorname{Im} \zeta_{1}\right)^{2}-q^{\prime 2}\left(\operatorname{Im} \zeta_{1}\right)^{2}\right]^{1 / 2}\right\}}
\end{gathered}
$$

2. Die Ungleichungen (IV.1) und (IV.2) zeigen: $M_{1}$ und $M_{2}$ besitzen Randwerte

$$
M_{1}\left(\zeta_{1}, \zeta, \zeta_{3}+i 0 ; \varphi_{q}\right), \quad M_{2}\left(\zeta_{1}, \zeta, \zeta_{3}-i 0 ; \varphi_{q}\right)
$$

in $\mathscr{S}^{\prime}\left(\mathbb{R}^{4}\right)$, und diese Randwerte sind analytische Funktionen in $\left(\zeta_{1}, \zeta\right) \in \mathscr{T}^{\prime} \times \mathbb{C}^{4}$.

$$
H\left(\zeta_{1}, \zeta, \xi_{3}\right):=M_{1}\left(\zeta_{1}, \zeta, \xi_{3}+i 0 ; \varphi_{q}\right)-M_{2}\left(\zeta_{1}, \zeta, \xi_{3}-i 0 ; \varphi_{q}\right)
$$

ist also in $\mathscr{T}^{\prime} \times \mathbb{C}^{4}$ analytisch. Sei $\xi_{3}^{2}<0$ und $\zeta_{1} \in \mathscr{T}^{+}$, dann ist

$$
H\left(\zeta_{1}, \zeta, \xi_{3}\right)=M_{11}\left(\zeta_{1}, \zeta, \xi_{3}+i 0 ; \varphi_{q}\right)-M_{12}\left(\zeta_{1}, \zeta, \zeta_{3}-i 0 ; \varphi_{q}\right)=0
$$

infolge der Lokalität nach G1. (II.5); folglich ist $H\left(\zeta_{1}, \zeta, \xi_{3}\right) \equiv 0$ in $\left(\zeta_{1}, \zeta\right) \in \mathscr{T}^{\prime} \times \mathbb{C}^{4}$ für $\xi_{3}^{2}<0$. Wie oben erhalten wir schließlich eine gemeinsame analytische Fortsetzung von $M_{1}$ und $M_{2}$ und damit von $M_{j k}\left(\zeta_{1}, \zeta, \zeta_{3} ; \varphi_{q}\right), j, k=1,2$ nach $\mathscr{T}^{\prime} \times \mathbb{C}^{4} \times \mathscr{T}^{\prime}$. Wie beim Nachweis der Ungleichung (IV.1) folgt aus (IV.1) und (IV.2) die Abschätzung

$$
\left|M\left(\zeta_{1}, \zeta, \zeta_{3} ; \varphi_{q}\right)\right| \leqq K_{\zeta, \varphi_{q}} \hat{\psi}(z) \psi^{*}\left(\zeta_{1}\right) \psi\left(\zeta_{3}\right) e^{\chi\left(q^{\prime}, \operatorname{Im} \zeta_{1}, \operatorname{Im} \zeta_{3}\right)}
$$

für $M$. Dabei ist $\psi(\zeta)=c|(\zeta, \zeta)|^{N}\left(1+\frac{1}{d_{\zeta}}\right)^{M}$ aus $\psi_{1}$ und $\psi_{2}$ ebenso konstruiert wie $\psi^{*}$ aus $\psi_{1}^{*}$ und $\psi_{2}^{*}$.

3. Die Trägereigenschaften von $M\left(\zeta_{1}, \zeta, \zeta_{3} ; \varphi_{q}\right)$ bezüglich $q$ sind nach dem Identitätssatz für holomorphe Funktionen dieselben wie die der $M_{j k}\left(\zeta_{1}, \zeta, \zeta_{3} ; \varphi_{q}\right)$

Nach diesen Vorbereitungen ist es einfach, eine analytische Fortsetzung der Wightman-Funktionen $M_{j k}$ durch ein Integral über die oben eingeführte Funktion $M\left(\zeta_{1}, \zeta, \zeta_{3} ; \varphi_{q}\right)$ anzugeben. Wir wollen, wie es die bisherige Konstruktion nahelegt, diese analytische Fortsetzung durch

$$
M_{\varphi}\left(\zeta_{1}, \zeta, \zeta_{3}\right):=\int M\left(\zeta_{1}, \zeta, \zeta_{3} ; \varphi_{q}\right) d^{4} q
$$


erklären. Eine hinreichende Bedingung dafür, daß durch (IV.3) eine in einem noch zu bestimmenden Bereich $\Omega$ analytische Funktion definiert wird, ist, daß das Integral in (IV.3) für jede kompakte Teilmenge $k \subset \Omega$ (absolut) gleichmäßig konvergiert. Eine hinreichende Bedingung für diese Konvergenz gewinnen wir aus den Wachstumseigenschaften nach Satz IV.1. Danach ist nämlich für $\zeta \in \mathscr{T}^{+}$

mit

$$
\left|M\left(\zeta_{1}, \zeta, \zeta_{3} ; \varphi_{q}\right)\right| \leqq\|\varphi\| \hat{\psi}(z) \psi^{*}\left(\zeta_{1}\right) \psi\left(\zeta_{3}\right) e^{-\left(q^{\prime \prime}, \eta\right)+\chi\left(q^{\prime}, \eta_{1}, \eta_{3}\right)}
$$

$$
\begin{gathered}
\|\varphi\|=\sup _{p}|\varphi(p)|, \quad \operatorname{Tr} \varphi_{q} \subset\left(q^{\prime \prime}+\overline{V^{+}}\right) \cap\left(q^{\prime}-\overline{V^{+}}\right), \\
q^{\prime}=q+\varepsilon, \quad q^{\prime \prime}=q-\varepsilon, \quad \eta_{j}=\operatorname{Im} \zeta_{j} .
\end{gathered}
$$

Der Identitätssatz für holomorphe Funktionen liefert:

$$
\varphi \rightarrow M\left(\zeta_{1}, \zeta, \zeta_{3} ; \varphi\right)
$$

ist linear in $\varphi \in \mathscr{C}_{c}^{0}\left(\mathbb{R}^{4}\right):=\left\{f: \mathbb{R}^{4} \rightarrow \mathbb{C} \mid f\right.$ stetig, Tr. $f$ kompakt $\}$. Die Ungleichung (IV.4) zeigt:

Ist $k\left(\mathscr{T}^{\prime} \times \mathscr{T}^{+} \times \mathscr{T}^{\prime}\right.$ eine kompakte Teilmenge, so haben wir in $\varphi \rightarrow M\left(\zeta_{1}, \zeta, \zeta_{3} ; \varphi\right),\left(\zeta_{1}, \zeta, \zeta_{3}\right) \in k$ eine gleichgradig beschränkte Familie stetiger Linearformen auf $\mathscr{C}_{c}^{0}\left(\mathbb{R}^{4}\right)$, also Radonsche Maße

$$
\begin{aligned}
M\left(\zeta_{1}, \zeta, \zeta_{3} ; \varphi\right) & \equiv \int \varphi(p) d M_{\zeta_{1}, \zeta, \zeta_{3}}(p) \\
M\left(\zeta_{1}, \zeta, \zeta_{3} ; \varphi_{q}\right) & =\left(M_{\zeta_{1}, \zeta, \zeta_{3}} * \varphi\right)(q) .
\end{aligned}
$$

Setzen wir nun

$$
\begin{gathered}
\lambda_{p}\left(\eta, \eta_{1}, \eta_{3}\right):=-(p, \eta)+\chi\left(p, \eta_{1}, \eta_{3}\right) \\
=-(p, \eta)+\frac{1}{2} \sum_{j=1,3} \max \left\{\mid\left(p, \eta_{j}\right),\left[\left(p, \eta_{j}\right)^{2}-p^{2} \eta_{j}^{2}\right]^{\frac{1}{2}}\right\} \\
\lambda\left(\eta, \eta_{1}, \eta_{3}\right):=\sup _{\substack{p \in \overline{V^{+}} \\
\|p\|=1}}\left\{\lambda_{p}\left(\eta, \eta_{1}, \eta_{3}\right)\right\}
\end{gathered}
$$

und

$$
\Omega:=\left\{\left(\zeta_{1}, \zeta, \zeta_{3}\right) \in \mathscr{T}^{\prime} \times \mathscr{T}^{+} \times \mathscr{T}^{\prime} \mid \lambda\left(\operatorname{Im} \zeta, \operatorname{Im} \zeta_{1}, \operatorname{Im} \zeta_{3}\right)<0\right\},
$$

dann folgt aus (IV.4) für kompakte Teilmengen $k \subset \Omega$ die absolute gleichmäßige Konvergenz von $\int M\left(\zeta_{1}, \zeta, \zeta_{3} ; \varphi_{q}\right) d^{4} q$ auf $k$, denn für $\left(\zeta_{1}, \zeta, \zeta_{3}\right) \in k$ ist

$$
\begin{aligned}
& \int\left|M\left(\zeta_{1}, \zeta, \zeta_{3} ; \varphi_{q}\right)\right| d^{4} q \leqq\|\varphi\| \hat{\psi}(z) \psi^{*}\left(\zeta_{1}\right) \psi\left(\zeta_{3}\right) \int_{-\varepsilon+\overline{V^{+}}} e^{-\left(q^{\prime}, \eta\right)+\chi\left(q^{\prime}, \eta_{1}, \eta_{3}\right)} d^{4} q \\
& =\|\varphi\| \hat{\psi}(z) \psi^{*}\left(\zeta_{1}\right) \psi\left(\zeta_{3}\right) e^{2(\varepsilon, \eta)} \frac{\int_{\overline{V^{+}}} e^{\lambda_{p}\left(\eta, \eta_{1}, \eta_{3}\right)} d^{4} p}{\quad \leqq}{ }^{4}\left\|\hat{\psi}(z) \psi^{*}\left(\zeta_{1}\right) \psi\left(\zeta_{3}\right) \frac{e^{2(\varepsilon, \eta)} C_{4}}{\left[\lambda\left(\eta, \eta_{1}, \eta_{3}\right)\right]^{4}} \leqq\right\| \varphi \| \hat{\psi}(z) \frac{e^{2\left(\varepsilon, \eta_{k}\right)} \psi_{k}^{*} \psi_{k} C_{4}}{\lambda_{k} 4}<\infty
\end{aligned}
$$


mit einer Konstanten $C_{4}$, die der Oberfläche der Einheitssphäre $S^{3}$ im $\mathbb{R}^{4}$ proportional ist, und $\psi_{k}^{*}, \psi_{k}, \lambda_{k}, e^{2\left(\varepsilon, \eta_{k}\right)}$ sind die Maxima der entsprechenden Funktionen auf $k$. Also ist das Radon-Maß $d M_{\zeta_{1}, \zeta, \zeta_{3}}(p)$ für $\left(\zeta_{1}, \zeta, \zeta_{3}\right) \in \Omega$ über $\overline{V^{+}}$integrierbar, und $M_{\varphi}\left(\zeta_{1}, \zeta, \zeta_{3}\right)$ ist in $\Omega$ analytisch.

Die obige Abschätzung von $M_{\varphi}\left(\zeta_{1}, \zeta, \zeta_{3}\right)=\int M\left(\zeta_{1}, \zeta, \zeta_{3} ; \varphi_{q}\right) d^{4} q$ kann verbessert werden (private Mitteilung von Prof. Borchers): Setze

mit

$$
\eta_{j}^{\prime}:=\left\{\begin{array}{cc}
\left(\operatorname{sign} \eta_{j}^{0}\right) \eta_{j}, & \eta_{j}^{0} \geqq 0 \\
\tilde{\eta}_{j}, & \eta_{j}^{2}<0
\end{array}\right.
$$

$$
\tilde{\eta}_{j}\left[\left(\eta, \eta_{j}\right)^{2}-\eta^{2} \eta_{j}^{2}\right]^{\frac{1}{2}}:=-\eta_{j}^{2} \eta+\left(\eta, \eta_{j}\right) \eta_{j} .
$$

Es ist $\eta_{j}^{\prime}$ stetig bei $\eta_{j}^{2}=0$ und $\tilde{\eta}_{j} \in V^{+}, \tilde{\eta}_{j}^{2}=-\eta_{j}^{2}$. Für $\eta_{j}^{2}<0$ sei

mit

$$
p_{\perp}=p-\alpha_{1} \eta-\alpha_{2} \eta_{j}
$$

$$
\begin{aligned}
& \alpha_{1}\left[\left(\eta, \eta_{j}\right)^{2}-\eta_{j}^{2} \eta^{2}\right]=\left(\eta_{j}, p\right)\left(\eta, \eta_{j}\right)-(\eta, p) \eta_{j}^{2} \\
& \alpha_{2}\left[\left(\eta, \eta_{j}\right)^{2}-\eta_{j}^{2} \eta^{2}\right]=(\eta, p)\left(\eta, \eta_{j}\right)-\left(\eta_{j}, p\right) \eta^{2}
\end{aligned}
$$

der zu $\eta$ und $\eta_{j}$ senkrechte Anteil von $p$.

Wegen $\eta \in V^{+}$ist $p_{\perp}^{2}=p^{2}-\alpha_{1}(p, \eta)-\alpha_{2}\left(p, \eta_{j}\right)<0$ für $p \in V^{+}$, und es gilt

$$
\left(p, \eta_{j}\right)^{2}-p^{2} \eta_{j}^{2}=\left(p, \tilde{\eta}_{j}\right)^{2}-p_{\perp}^{2} \eta_{j}^{2}<\left(p, \tilde{\eta}_{j}\right)^{2}
$$

für $\eta_{j}^{2}<0$. Folglich ist

$$
\lambda_{p}\left(\eta, \eta_{1}, \eta_{3}\right) \leqq-(p, \xi)
$$

mit

$$
\xi=\xi\left(\eta, \eta_{1}, \eta_{3}\right):=\eta-\frac{1}{2}\left(\eta_{1}^{\prime}+\eta_{3}^{\prime}\right) .
$$

In (IV.8) steht das Gleichheitszeichen nur dann, wenn $\eta_{j}^{2} \geqq 0$ für $j=1$ und $j=3$. Fordern wir nun $\xi \in V^{+}$, so ist

$$
\int_{V^{+}} e^{\lambda_{p}\left(\eta, \eta_{1}, \eta_{3}\right)} d^{4} p \leqq \int_{V^{+}} e^{-(p, \xi)} d^{4} p=\frac{c}{\left[\xi^{2}\right]^{2}}<\infty,
$$

d. h. auch die Forderung $\xi \in V^{+}$garantiert, daß das $M a ß d M_{\xi, \zeta, \zeta_{3}}(p)$ über $\overline{V^{+}}$integrierbar und damit $M_{\varphi}\left(\zeta_{1}, \zeta, \zeta_{3}\right)$ analytisch ist.

Wenn $\xi \in V^{+}$, so ist

$$
\lambda\left(\eta, \eta_{1}, \eta_{3}\right) \leqq \sup _{p \in \overline{V^{+}} \cap S^{3}}\{-(p, \xi)\}=-d_{\xi}<0
$$

also $\left(\zeta_{1}, \zeta, \zeta_{3}\right) \in \Omega$, aber die Umkehrung gilt nicht: Es gibt Punkte mit $\lambda\left(\eta, \eta_{1}, \eta_{3}\right)<0$ und $\xi=\xi\left(\eta, \eta_{1}, \eta_{3}\right) \notin V^{+}$. Zusammen erhalten wir folgende 
Abschätzung:

$$
\left|M_{\varphi}\left(\zeta_{1}, \zeta, \zeta_{3}\right)\right| \leqq\|\varphi\| \hat{\psi}(z) \psi^{*}\left(\zeta_{1}\right) \psi\left(\zeta_{3}\right) \frac{C e^{2(\eta, \varepsilon)}}{\left[\min \left\{\xi^{2}, \lambda^{2}\right\}\right]^{2}}
$$

Für ${ }^{ \pm} \eta_{1},{ }^{ \pm} \eta_{3} \in \overline{V^{+}}$ist $\lambda=-d_{\xi}$, also $\min \left\{\xi^{2}, \lambda^{2}\right\}=\xi^{2}$.

Dieses führt zu dem

Satz IV.2. Wird $M\left(\zeta_{1}, \zeta, \zeta_{3} ; \varphi_{q}\right)$ wie in Satz IV.1 erklärt, so definiert

$$
M\left(\zeta_{1}, \zeta, \zeta_{3}\right):=\int M\left(\zeta_{1}, \zeta, \zeta_{3} ; \varphi_{q}\right) d^{4} q
$$

eine eindeutige $\mathscr{L}_{+}^{\uparrow}$-invariante, mindestens in $\Omega$ analytische Funktion. $M\left(\zeta_{1}, \zeta_{2}, \zeta_{3}\right)$ ist unabhängig von der Wahl der Funktion $\varphi$ mit den Eigenschaften (II.10) und setzt die vier Wightman-Funktionen $M_{j k}$ nach $\Omega$ fort. Der Bereich $\Omega$ ist durch (IV.7) erklärt. $M\left(\zeta_{1}, \zeta, \zeta_{3}\right)$ wächst nicht stärker als folgende Ungleichung zuläßt:

$$
\left|M\left(\zeta_{1}, \zeta, \zeta_{3}\right)\right| \leqq \frac{C_{0}\left(\eta^{0}\right)^{4} \psi^{*}\left(\zeta_{1}\right) \psi\left(\zeta_{3}\right)}{\left[\min \left\{\xi^{2}, \lambda^{2}\right\}\right]^{2}}
$$

Beweis. 1. Daß $M_{\varphi}\left(\zeta_{1}, \zeta, \zeta_{3}\right)=\int M\left(\zeta_{1}, \zeta, \zeta_{3} ; \varphi_{q}\right) d^{4} q$ mindestens in $\Omega$ analytisch ist, wurde bereits oben gezeigt.

2. Später untersuchen wir die Menge $\Omega$ etwas genauer und zeigen u.a., daß $\Omega$ ein Gebiet ist, welches die vier Tuben enthält. Daher können wir folgendermaßen schließen:

Die Sätze (IV.1) und (II.8) implizieren:

$$
\begin{aligned}
& \left.M_{\varphi}\left(\zeta_{1}, \zeta, \zeta_{3}\right)\right|_{\Omega j k}=\left.\int M\left(\zeta_{1}, \zeta, \zeta_{3} ; \varphi_{q}\right)\right|_{\Omega j k} d^{4} q \\
& \quad=\int M_{j k}\left(\zeta_{1}, \zeta, \zeta_{3} ; \varphi_{q}\right) d^{4} q=M_{j k}\left(\zeta_{1}, \zeta, \zeta_{3}\right) .
\end{aligned}
$$

Diese Relation garantiert mit Hilfe des Identitätssatzes für holomorphe Funktionen, daß die durch $\int M\left(\zeta_{1}, \zeta, \zeta_{3} ; \varphi_{q}\right) d^{4} q$ erklärte Funktion unabhängig von der speziellen Wahl der Funktion $\varphi$ mit den Eigenschaften (II.10) ist und in gleicher Weise die $\mathscr{L}_{+}^{\uparrow}$-Invarianz der Funktion $M\left(\zeta_{1}, \zeta, \zeta_{3}\right)$, denn die $M_{j k}\left(\zeta_{1}, \zeta, \zeta_{3}\right)$ sind $\mathscr{L}_{+}^{\uparrow}$-invariant.

3. Infolge $\int \varphi(p) d^{4} p=1$ und $\operatorname{Tr} \varphi \subset\left(-\varepsilon+V^{+}\right) \cap\left(\varepsilon-V^{+}\right)$ist $\|\varphi\|$ proportional $\mathrm{zu} \varepsilon^{\prime 4}\left(\varepsilon=\left(\sqrt{2} \varepsilon^{\prime}, \underline{0}\right)\right)$. Nach 2 . können wir für beliebige aber feste $\left(\zeta_{1}, \zeta, \zeta_{3}\right) \in \Omega \varphi$ so wählen, daß $2(\varepsilon, \eta)=2 \varepsilon^{\prime} \eta^{0}=4$ gilt. Dann ist $\|\varphi\| e^{2(\varepsilon, \eta)}=$ const $\cdot\left(\eta^{0}\right)^{4}$.

4. Die $z$-Abhängigkeit der Zustände $\Phi_{j k}(z, \zeta)$ trägt zum Wert der Matrixelemente $M_{j k}$ nach (II.6) infolge der Translationsinvarianz nichts bei. Daher hängt auch die analytische Fortsetzung $M \operatorname{der} M_{j k}$ nicht von $z \in \mathscr{T}^{+}$ab. Wir können also für die Abschätzung des Wachstums von $M z_{0} \in \mathscr{T}^{+}$beliebig aber fest wählen, so daß $\hat{\psi}(z)=\hat{\psi}\left(z_{0}\right)=$ const. Mit 3 . folgt aus (IV.9) die Ungleichung (IV.10). 
Der Beweis ist erst vollständig, wenn $\Omega$ als Gebiet erkannt worden ist, welches die vier Tuben $\Omega_{j k}$ enthält. Das wird im folgenden Satz nachgeholt:

Satz IV.3. Sei $\Omega:=\left\{\left(\zeta_{1}, \zeta, \zeta_{3}\right) \in \mathscr{T}^{\prime} \times \mathscr{T}^{+} \times \mathscr{T}^{\prime} \mid \lambda\left(\operatorname{Im} \zeta_{,} \operatorname{Im} \zeta_{1}, \operatorname{Im} \zeta_{3}\right)<0\right\}$ mit der durch (IV.6) erklärten Funktion $\lambda$. Dann gilt:

(a) $\Omega$ enthält die Tuben $\Omega_{j k}$ und ist echt größer als die Vereinigung dieser Tuben.

(b) $\Omega$ ist ein Gebiet.

(c) $\Omega$ ist $\mathscr{L}_{+}^{\uparrow}$-invariant.

(d) $\Omega$ ist ein Holomorphiegebiet.

Beweis. (a) Sei z. B. $\left(\zeta_{1}, \zeta, \zeta_{3}\right) \in \Omega_{11}$, d. h. $\left(\zeta_{1}, \zeta-\frac{1}{2}\left(\zeta_{1}+\zeta_{3}\right), \zeta_{3}\right) \in \mathscr{T}_{3}^{+}$, folglich $\zeta \in \mathscr{T}^{+}$und

$$
\lambda\left(\operatorname{Im} \zeta, \operatorname{Im} \zeta_{1}, \operatorname{Im} \zeta_{3}\right)=-d_{\operatorname{Im}\left(\zeta-\frac{1}{2}\left(\zeta_{1}+\zeta_{3}\right)\right)}<0,
$$

wenn $d_{y}$ wieder den euklidischen Abstand des Punktes $y \in V^{+}$von $\mathbb{R}^{4} \backslash V^{+}$bezeichnet; also $\left(\zeta_{1}, \zeta, \zeta_{3}\right) \in \Omega$. Bei den übrigen "Tuben" $\Omega_{j k}$ geht der Nachweis entsprechend, und damit gilt:

denn

$$
\bigcup_{j, k=1,2} \Omega_{j k} \subset \Omega .
$$

$\Omega$ enthält Punkte, die nicht in $\bigcup_{j, k=1,2} \Omega_{j k}$ liegen. Z. B. ist

$$
\mathscr{I} \times \mathscr{T}^{+} \times \mathscr{I}=\left(\mathscr{T}^{\prime} \cap \mathbb{R}^{4}\right) \times \mathscr{T}^{+} \times\left(\mathscr{T}^{\prime} \cap \mathbb{R}^{4}\right) \subset \Omega \backslash \bigcup_{j, k=1,2} \Omega_{j k},
$$

$$
\lambda(\operatorname{Im} \zeta, 0,0)=-d_{\operatorname{Im} \zeta}<0,
$$

für reelle $\xi_{j}$. Ebenso leicht ist es zu zeigen, daß das Holomorphiegebiet

$$
\begin{aligned}
\Omega_{\alpha}:= & \left\{\left(\zeta_{1}, \zeta, \zeta_{3}\right) \in \mathscr{T}^{\prime} \times \mathscr{T}^{+} \times \mathscr{T}^{\prime} \mid \alpha\left(\operatorname{Im} \zeta_{1}, \operatorname{Im} \zeta, \operatorname{Im} \zeta_{3}\right)<0\right\} \\
& \alpha\left(\eta_{1}, \eta, \eta_{3}\right):=-\eta^{0}+|\eta|+\sum_{j=1,3} \max \left\{\left|\eta_{j}^{0}\right|,\left|\underline{\eta}_{j}\right|\right\}
\end{aligned}
$$

echt in $\Omega$ enthalten ist.

(b) Wir zeigen, daß $\Omega$ wegzusammenhängend ist. Nach (a) ist insbesondere die zusammenhängende Menge $\mathscr{I} \times \mathscr{T}^{+} \times \mathscr{I}$ in $\Omega$ enthalten. Nun gibt es stetige Wege $\left(\zeta_{1}(t), \zeta, \zeta_{3}(t)\right), 0 \leqq t \leqq 1$, die einen beliebigen Punkt aus $\mathscr{T}^{\prime} \times \mathscr{T}^{+} \times \mathscr{T}^{\prime}$ mit Punkten $\left(\xi_{1}^{\prime}, \zeta, \xi_{3}^{\prime}\right)$ aus $\mathscr{I} \times \mathscr{T}^{+} \times \mathscr{I}$ derart in $\mathscr{T}^{\prime} \times \mathscr{T}^{+} \times \mathscr{T}^{\prime}$ verbinden, daß

$$
\begin{aligned}
\lambda\left(\operatorname{Im} \zeta, \operatorname{Im} \zeta_{1}(t), \operatorname{Im} \zeta_{3}(t)\right) & \leqq \lambda\left(\operatorname{Im} \zeta, \operatorname{Im} \zeta_{1}\left(t^{\prime}\right), \operatorname{Im} \zeta_{3}\left(t^{\prime}\right)\right) \\
& \leqq \lambda\left(\operatorname{Im} \zeta, \operatorname{Im} \zeta_{1}, \operatorname{Im} \zeta_{3}\right),
\end{aligned}
$$

gilt für $0 \leqq t^{\prime}<t \leqq 1$, d. h. ist $\left(\zeta_{1}, \zeta, \zeta_{3}\right) \in \Omega$, so verläuft dieser Weg in $\Omega$.

Dieses folgt aus der Tatsache, da $\beta$ jeder Punkt $\zeta \in \mathscr{T}^{\prime}$ durch einen stetigen Weg $\zeta(t)$ in $\mathscr{T}^{\prime}$ mit Punkten $\xi_{0} \in \mathscr{I}$ so verbunden werden kann, 
$\operatorname{da}(\eta(t)=\operatorname{Im} \zeta(t))$

$$
\begin{aligned}
& \max \left\{|(p, \eta(t))|,\left[(p, \eta(t))^{2}-p^{2}(\eta(t))^{2}\right]^{\frac{1}{2}}\right\} \\
& \quad \leqq(1-t) \max \left\{|(p, \eta)|,\left[(p, \eta)^{2}-p^{2} \eta^{2}\right]^{\frac{1}{2}}\right\}
\end{aligned}
$$

gilt.

(c) $\Omega$ ist $\mathscr{L}_{+}^{\uparrow}$-invariant:

$\mathrm{Zu}$ zeigen bleibt nur, daß die Tube

$$
\Omega_{\lambda}:=\left\{\left(\zeta_{1}, \zeta, \zeta_{3}\right) \in \mathbb{C}^{3 \cdot 4} \mid \lambda\left(\operatorname{Im} \zeta, \operatorname{Im} \zeta_{1}, \operatorname{Im} \zeta_{3}\right)<0\right\}
$$

$\mathscr{L}_{+}^{\uparrow}$-invariant ist. Sei $\left(\zeta_{1}, \zeta, \zeta_{3}\right) \in \Omega_{\lambda}$ und $\Lambda \in \mathscr{L}_{+}^{\uparrow}$; dann ist

$$
\begin{gathered}
\lambda\left(\operatorname{Im} \Lambda \zeta, \operatorname{Im} \Lambda \zeta_{1}, \operatorname{Im} \Lambda \zeta_{3}\right)=\sup _{p \in \overline{V^{+}} \cap S^{3}} \cdot\left\{\lambda_{\Lambda^{-1} p}\left(\operatorname{Im} \zeta, \operatorname{Im} \zeta_{1}, \operatorname{Im} \zeta_{3}\right)\right\} \\
\leqq \min _{q \in \Lambda^{-1}\left(\overline{V^{+}} \cap S^{3}\right)}\{\|q\|\} \cdot \lambda\left(\operatorname{Im} \zeta, \operatorname{Im} \zeta_{1}, \operatorname{Im} \zeta_{3}\right)<0
\end{gathered}
$$

also $\left(\Lambda \zeta_{1}, \Lambda \zeta, \Lambda \zeta_{3}\right) \in \Omega_{\lambda}$.

(d) Um zu beweisen, daß $\Omega$ ein Holomorphiegebiet ist, schreiben wir

$$
\Omega=\Omega_{\lambda} \cap\left(\mathscr{T}^{\prime} \times \mathscr{T}^{+} \times \mathscr{T}^{\prime}\right), \quad \Omega_{\lambda}=\left(\bigcap_{p \in \frac{\bigcap^{+}}{V^{+}} S^{3}} \Omega_{\lambda p}\right)^{0}
$$

$\left(B^{0}=\right.$ offener Kern der Menge $\left.B\right)$ und beweisen, da

mit

$$
\Omega_{\lambda_{p}}:=\left\{\left(\zeta_{1}, \zeta, \zeta_{3}\right) \mid \lambda_{p}\left(\operatorname{Im} \zeta, \operatorname{Im} \zeta_{1}, \operatorname{Im} \zeta_{3}\right)<0\right\}
$$

$$
\lambda_{p}\left(\eta, \eta_{1}, \eta_{3}\right)=-(p, \eta)+\frac{1}{2} \sum_{j=1,3} \max \left\{\left|\left(p, \eta_{j}\right)\right|,\left[\left(p, \eta_{j}\right)^{2}-p^{2} \eta_{j}^{2}\right]^{\frac{1}{2}}\right\}
$$

ein Holomorphiegebiet ist. Nach ([11], p. 40) folgt daraus die Behauptung.

Dazu sei $t, t^{\prime} \geqq 0$ und $t+t^{\prime}=1$; dann ist

$$
\begin{aligned}
& \lambda_{p}\left(t \eta+t^{\prime} \eta^{\prime}, t \eta_{1}+t^{\prime} \eta_{1}^{\prime}, t \eta_{3}+t^{\prime} \eta_{3}^{\prime}\right) \leqq \lambda_{p}\left(t \eta, t \eta_{1}, t \eta_{3}\right) \\
& \quad+\lambda_{p}\left(t^{\prime} \eta^{\prime}, t^{\prime} \eta_{1}^{\prime}, t^{\prime} \eta_{3}^{\prime}\right)=t \lambda_{p}\left(\eta, \eta_{1}, \eta_{3}\right)+t^{\prime} \lambda_{p}\left(\eta^{\prime}, \eta_{1}^{\prime}, \eta_{3}^{\prime}\right),
\end{aligned}
$$

d. h. $\lambda_{p}$ ist eine konvexe Funktion und damit $\Omega_{\lambda_{p}}$ eine konvexe Tube, mithin ein Holomorphiegebiet ([11], p. 43).

Wir schließen mit einigen Bemerkungen:

1. Die Darstellung des Holomorphiegebietes $\Omega$ in den ursprünglichen Variablen $\left(z_{1}, z_{2}, z_{3}, z_{4}\right) \in \mathbb{C}^{4 \cdot 4}$ der Vierpunkt-Wightman-Funktion zeigt in einfacher Weise die Invarianz von $\Omega$ unter den Permutationen (1234), (2134), (1243) und (2143), die den vier Tuben entsprechen, die durch $\Omega$ vereinigt werden. Es ist nämlich

$$
\begin{aligned}
\hat{\Omega}= & \left\{\left(z_{1}, z_{2}, z_{3}, z_{4}\right) \mid z_{2}-z_{1} \in \mathscr{T}^{\prime}, z_{4}-z_{3} \in \mathscr{T}^{\prime},\right. \\
& \left.\frac{1}{2}\left(z_{3}+z_{4}-z_{1}-z_{2}\right) \in \mathscr{T}^{+}, \hat{\lambda}\left(z_{1}, z_{2}, z_{3}, z_{4}\right)<0\right\}
\end{aligned}
$$


mit

$$
\hat{\lambda}\left(z_{1}, z_{2}, z_{3}, z_{4}\right)=\sup _{p \in S^{3} \cap \overline{V^{+}}}\left\{\hat{\lambda}_{p}\left(z_{1}, z_{2}, z_{3}, z_{4}\right)\right\}
$$

$$
\begin{aligned}
& \hat{\lambda}_{p}\left(z_{1}, z_{2}, z_{3}, z_{4}\right)=-\frac{1}{2}\left(p, y_{3}+y_{4}-y_{1}-y_{2}\right) \\
& \quad+\frac{1}{2} \max \left\{\left|\left(p, y_{2}-y_{1}\right)\right|,\left[\left(p, y_{2}-y_{1}\right)^{2}-p^{2}\left(y_{2}-y_{1}\right)^{2}\right]^{\frac{1}{2}}\right\} \\
& \quad+\frac{1}{2} \max \left\{\left|\left(p, y_{4}-y_{3}\right)\right|,\left[\left(p, y_{4}-y_{3}\right)^{2}-p^{2}\left(y_{4}-y_{3}\right)^{2}\right]^{\frac{1}{2}}\right\}, \quad y_{j}=\operatorname{Im} z_{j} .
\end{aligned}
$$

2. Es wurde gezeigt: Die vier $W$-Funktionen $M_{j k}$ besitzen eine gemeinsame $\mathscr{L}_{+}^{\uparrow}$-invariante analytische Fortsetzung in ein $\mathscr{L}_{+}^{\uparrow}$-invariantes Holomorphiegebiet. Damit liegt genau die entsprechende Situation vor, die für das Analytizitätsgebiet einer einzigen Wightman-Funktion Anlaß zum BHW-Theorem ([13], p. 78) gab. So erhebt sich die Frage, welche Ergebnisse hier Untersuchungen im Sinne des BHW-Theorems haben könnten, bzw. welche zusätzlichen Aussagen man aus der nach dem BHW-Theorem bekannten $\mathscr{L}_{+}(\mathbb{C})$-Invarianz der gemeinsamen analytischen Fortsetzung der $W$-Funktionen in ein $\mathscr{L}_{+}(\mathbb{C})$-invariantes Gebiet gewinnen könnte. $\Omega$ ist offensichtlich und erwartungsgemäß nicht $\mathscr{L}_{+}(\mathbb{C})$ invariant.

Für die Anregung dieser Untersuchungen und für zahlreiche Hinweise und ermutigende Diskussionen möchte ich Herrn Prof. Dr. H. J. Borchers herzlich danken.

\section{Literatur}

1. Araki, H.: Einführung in die Axiomatische Quantenfeldtheorie, E.T.H. (1961-1962).

2. - Varenna Lectures XLV (1968), p. 65-96. Ed.: R. Jost. New York: Academic Press 1969.

3. Borchers, H.J.: In: F.Lurçat (Ed.): Applications of Mathematics to Problems in Theoretical Physics. New York: Gordon \& Breach 1967.

4. - Einführung in die Quantenfeldtheorie II. Vorlesung, Göttingen 1967/68.

5. Bros, J., Epstein, H., Glaser, V.: Commun. math. Phys. 6, 77 (1967).

6. Dixmier, J.: Les Algèbres d'operateurs dans l'espace hilbertien. Paris: Gauthier Villars 1956.

7. Epstein, H., Glaser, V., Martin, A.: Commun. math. Phys. 13, 257 (1969).

8. Fuks, B.A.: Introduction to the theory of analytic functions of several complex variables. Ann. Math. Soc. transl. (1963).

9. Haag, R., Schroer, B.: J. Math. Phys. 3, 248 (1962).

10. Hepp, K.: Helv. Phys. Acta 37, 639 (1964).

11. Hörmander, L.: An introduction to complex analysis in several variables. Princeton: van Nostrand 1966.

12. Hall, R., Wightman, A.S.: Mat. Fys. Medd. Danske Vid. Selsk. 31, No. 5 (1957).

13. Jost, R.: The general theory of quantized fields; American Mathematical Society 1965, Vol. IV.

14. Ruelle, D.: Helv. Phys. Acta 32, 135 (1959).

15. Sénéor, R.: Commun. math. Phys. 11, 242 (1968/69). 
16. Streater, R.F.: J. Math. Phys. 3, 256 (1962).

17. - Wightman, A.S.: PCT, spin and statistics, and all that. New York: Benjamin 1964.

18. Uhlmann, A.: Ann. Phys. 13, 453 (1961).

19. Vladimirov, V.S.: Methods of the theory of functions of several complex variables. Boston: MIT Press 1966.

20. Wightman, A. S.: Phys. Rev. 101, 860 (1956).

21. Schwartz, L.: Théorie des distributions, Vol, I, II. Paris: Hermann 1957, 1959.

\section{E. Brüning}

Theoretische Physik I

am Physikalischen Institut der Universität

D-8700 Würzburg, Röntgenring 8 